\title{
دراسة العلاقة السببية بين النمو الاقتصادي والإنفاق الحكومي في الجزائر، من خلال اختبار قانون فاجنر الإنفات خلال الفترة (1967-2017)
}

\section{The Study of the Causality Between the Economic Growth and Government Expenditure in Algeria,} Through Testing the Wagner's Law During the Period 1967-2017

\section{Ms. Atmania Khadra}

PhD. Student /University of Arabi Tbsi (Tebessa)/Algeria atm17man2015@gmail.com

\section{Dr. Ait Yahia Samir}

Lecturer "A"/ University of Arabi Tbsi (Tebessa)/ Algeria say082@yahoo.com

\author{
أ. عثمانية خصرة \\ طالبة دكتور اه/جامعة العربي التبسي (تبسة)/الجزائر
}

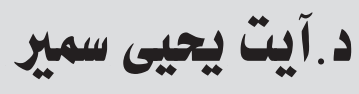

دكتور محاضر "أ"/جامعة العربي التبسي (تبسة)/الجزائر
Received: 1/ 12/ 2018, Accepted: 3/ 3/ 2019 DOI:

http: //journals.qou.edu/index.php/eqtsadia
تاريخ الاستلام: 1/ 12/ 2018م، تاريخ القبول: 3/ 3/ 2019 2019م.

E - ISSN: 2410 - 3349

P - ISSN: 2313 - 7592 
اتجـاه العلاقة يكون من النمو الاقتصادي إلى الإنفاق الحكومي

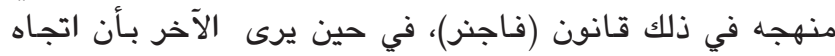

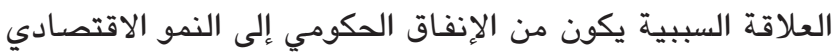

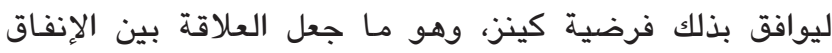

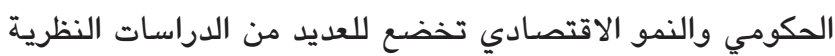

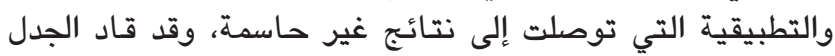

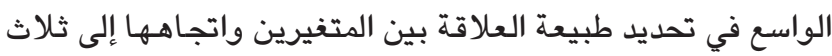

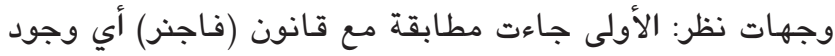

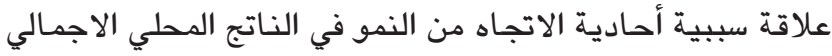

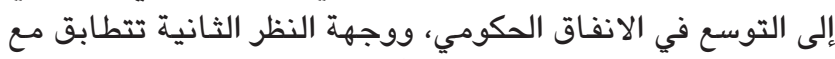

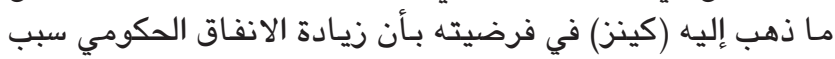

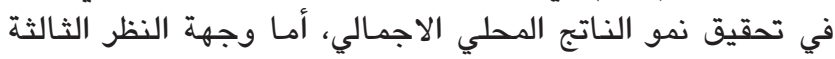

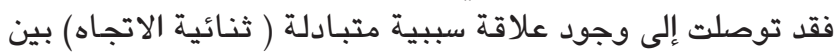

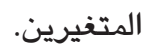

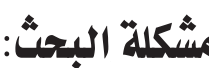

كان عدم التوافق في الآراء سواء في الأدبيات النظرية،

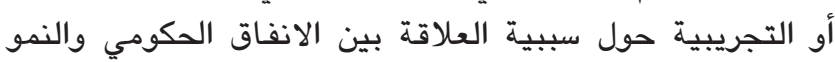

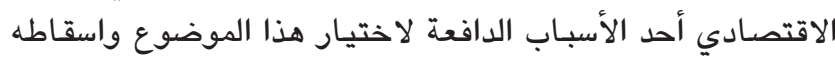

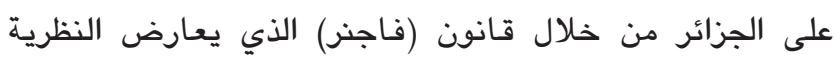

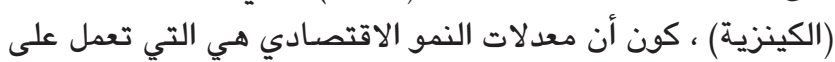
زيادة الإنفاق الحكومي للبلد وليس العكس.

تسعى الجزائر كغيرهـا من الدول إلى تحقيق معدلات مقبولة

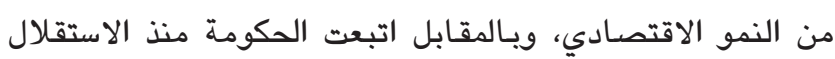
مخططات تنموية تعتمد على سياسات إنفاق حكومية توسعية في كل

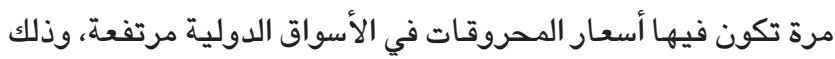

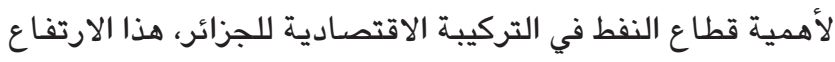

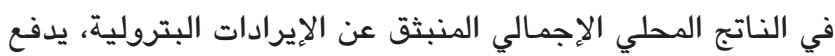

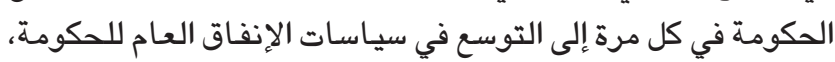

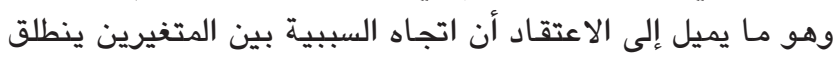

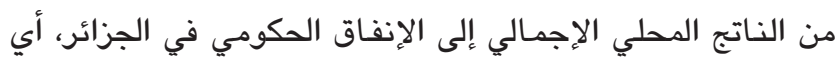

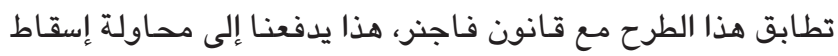

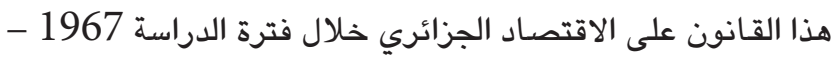

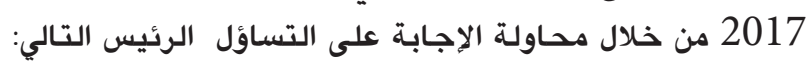
ما دلى صحة ومطابقة قانون فاجنر على الاقتصاد

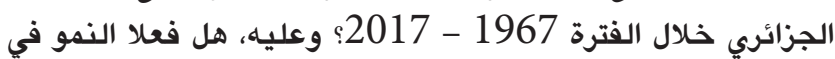
الناتج المحلي الإجمالي هو الذي أدى إلى زيـادة الإنفاق الحكومي في في في

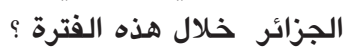

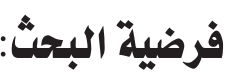

للإجابـة على التساؤل الرئيس السابق تمت صياغة الفرضية

الرئيسة التالية:

هناك علاقة سببية مستقرة وموجبة طويلة الأجل ويـاتجاه

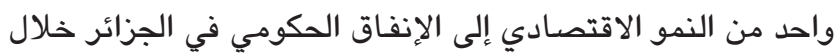

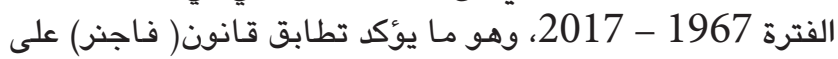
الاقتصـاد الجزائري.
تهدف هذه الورقة البحثية إلى محاولة تحليل العلاقة بين النمو الاقتصادي والإنفاق الحكومي في الجزائر خلال فلة الفي الفترة 1967 - 2017، وذلك بدراسة العلاقة السببية بين الإنئني المتغيرين باستعمال منهجية التكامل المشترك ونموذج تصدية الصديح الخطأ. وقد توصلت الدراسة إلى أن كلا السلسلتين مستقرتان في الفارق النارق

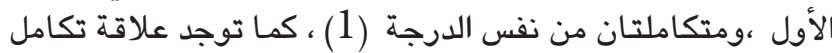

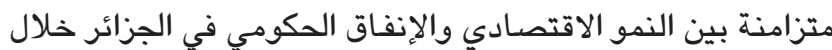
فترة الدراسة. ويعد اختبار سببية لفية الافتهادي

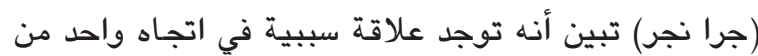

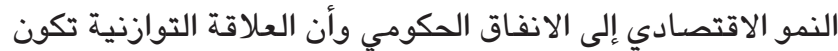
في المدى الطويل وهو ما يوافق قانون الانياق النكون (فاجنر).

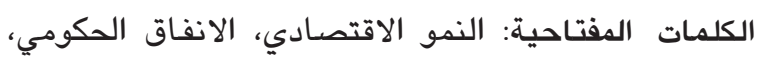
قانون فاجنر، الجزائر.

\section{Abstract:}

This paper attempts to analyze the relationship between economic growth and government expenditure in Algeria during the period 19672017- through studying the causality between these two variables. Using the Co-integration Regression and Error Correction Model, the study found that both series are stable at the first difference and are complementary at the same degree I(1), and there is a synchronous integration relationship between economic growth and government expenditure in Algeria. According to the Granger test, the study also found that there is a causality in a one-way direction, from economic growth to government expenditure, and the equilibrium relationship is in the long run, which is in accordance with Wagner s law.

Keywords: Economic Growth, Government Expenditure, Wagner's law, Algeria.

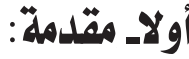

اختلفت المدارس الاقتصادية عبر الزمن حول أهمية دور

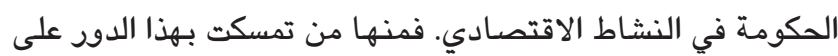

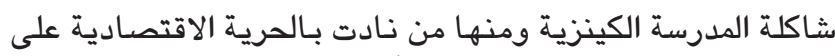

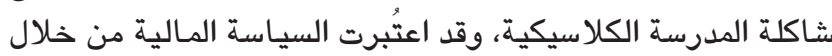

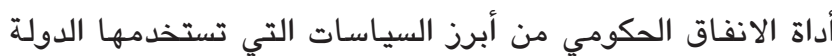

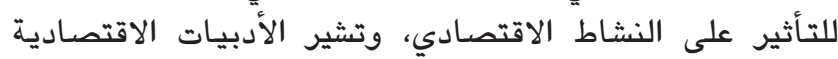

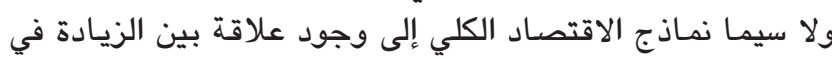

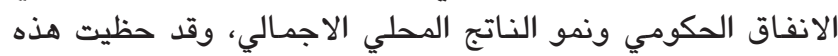

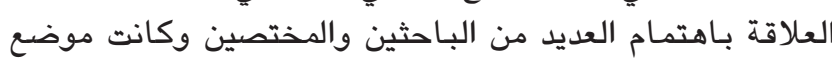

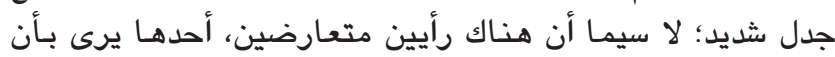


بين الإنفاق الحكومي الحقيقي والناتج المحلي الإجمالي في العراق

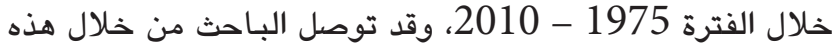

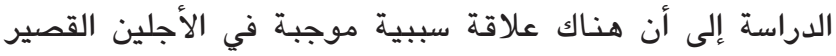

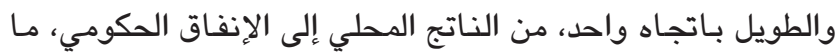
يدعم بشكل واضح تأثير قانون (فاجنر) في الاقتصـاد العراقي.

دراسة (Monogbe \& Okaho. 2016)

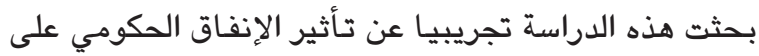

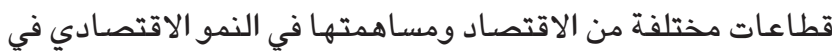

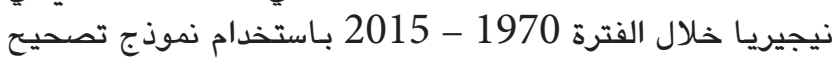

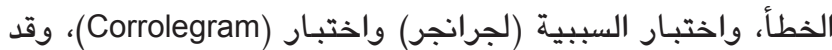

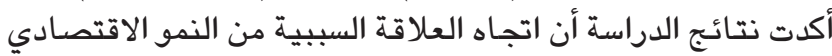

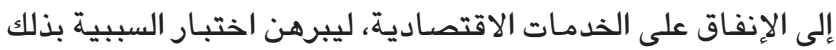

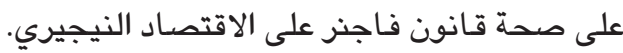

دراسة (Gizen Vzuner et al. 2017)

سعت هذه الدراسة للتحقق من صحة قانون فاجنر على القى الاقتصاد التركي خلال الفترة: 1975 - 2014 التونة باستخدام اختبار

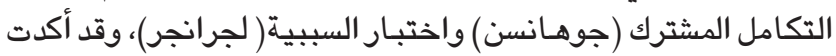

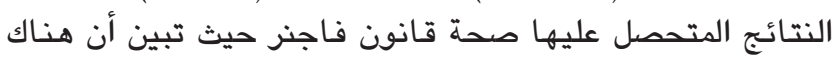

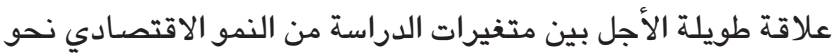

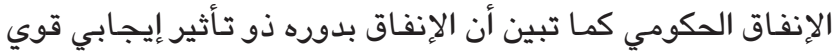

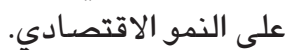

بوالكور نور الدين (2017)

سعت هذه الدراسة إلى تحليل العلاقة بين الإنفاق الحكومي

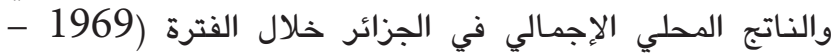

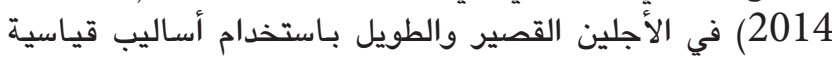
حديثة، كاختبار استقرارية السلاسل الزمنية والتكامل المشترك النترك

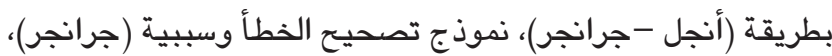

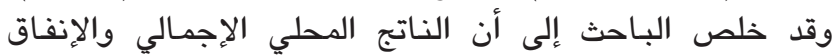

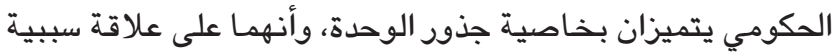
قصيرة وطويلة الأجل، تتجه من الناتج المحلي الإجمالي إلى الإنفاق التهاق

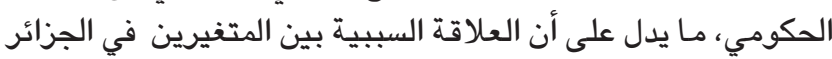
تتبع قانون فاجنر. - الإضافة التي جاءت بـها هذه الدراسة:

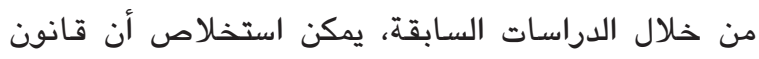

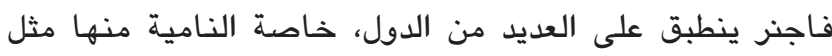

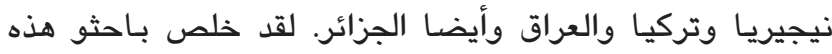

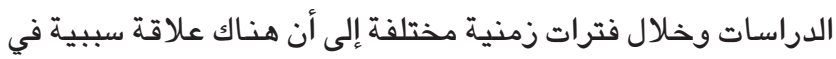

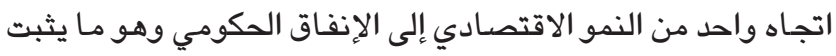

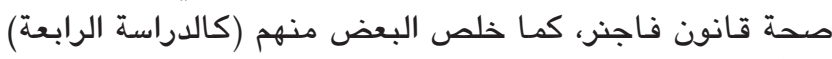

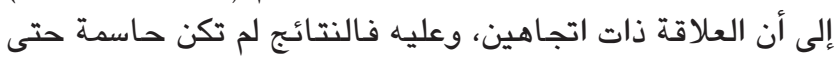

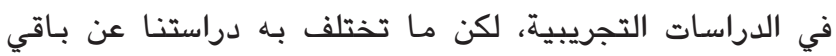
الدراسات هي النقاط التالية:

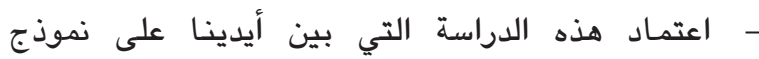

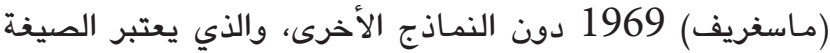

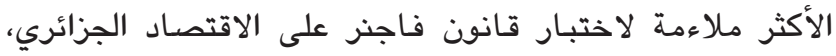

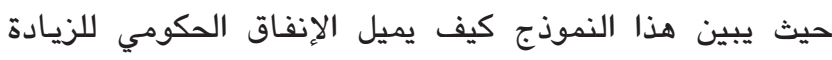

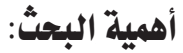

تأتي أهمية الدراسة كونها تتناول موضوعا يحتل مكانة

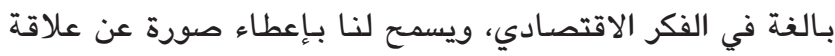
النمو الاقتصادي بالإنفاق الحكومي في الجزائر من خلال الإلهي الأهمية علية

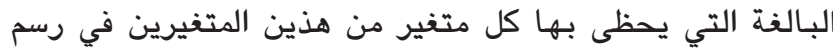

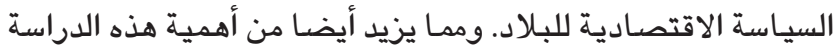

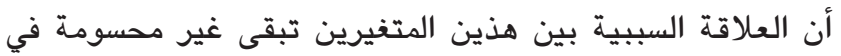
معظم الدراسات سواء النظرية منها أو حتى التجريبية.

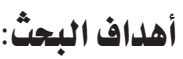

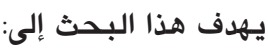

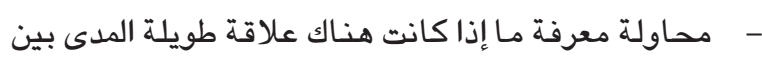

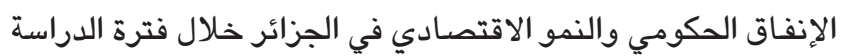
من خلال اختبار التكامل المشترك.

- - الكشف عن طبيعة العلاقة السببية بين متغيري الدراسة السابقين من خلال اختبار سببية (جرانجر) واتجاهـها.

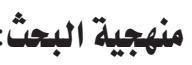

للإجابة على التساؤل السابق وللتوصل إلى ما يؤكد أو ينفي

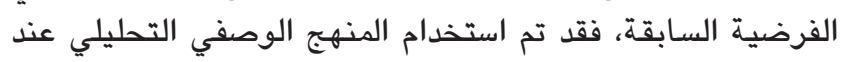

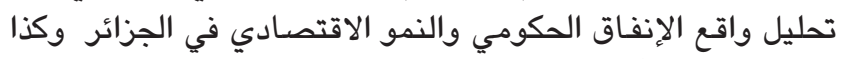

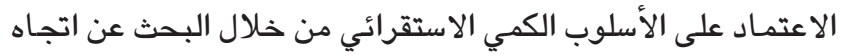

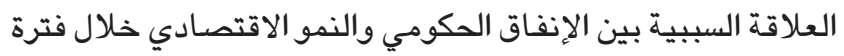

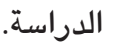

\section{حلدود البحث: اكث}

يقتصر الإطار المكاني للدراسة على دولة الجزائر، أما الإطار

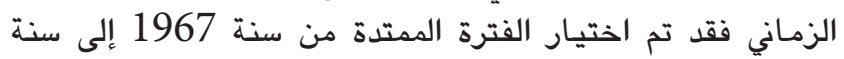

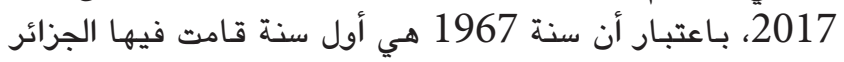
المستقلة بأول مخطط ثلاثي يعتمد على الإنفاق أنس الحكومي بشكل

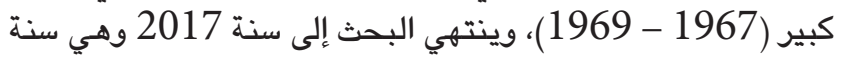

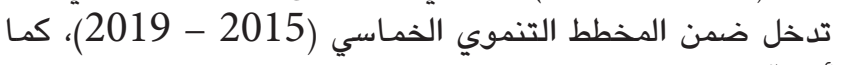
أنها آخر سنة تتوفر فيها إحصائيات الدراسة.

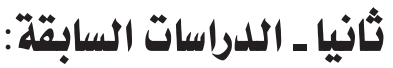

دراسة محمد أدريوش وعبد القادر (2012)

سعت هذه الدراسة إلى إثبات صحة قانون (فاجنر) في الجزائر

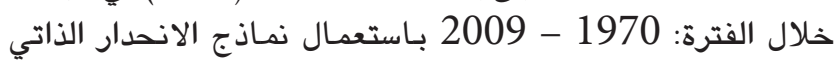

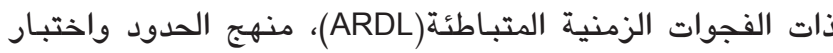
Toda and Yammamoto

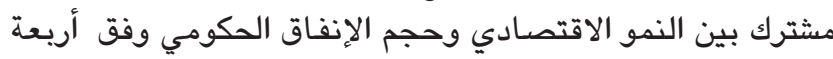

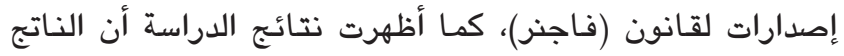

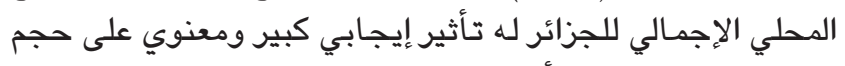

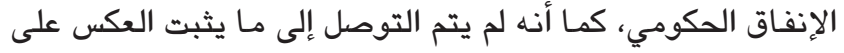

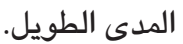

دراسة كريم سالم (2012) - (2015)

تهدف هذه الدراسة إلى محاولة ايجاد طبيعة، واتجاه العلاقة 


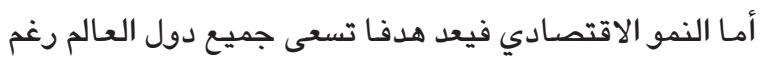

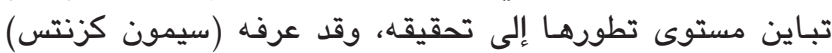

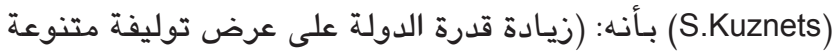

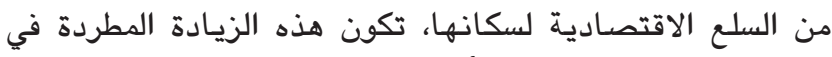
القدرة الإنتاجية مبنية على أساس التقدم التكنولوجية التوني والتعديلات

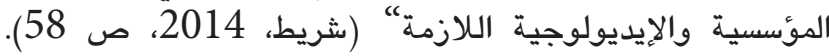

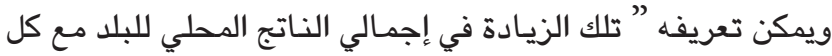

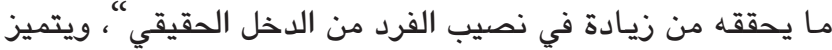

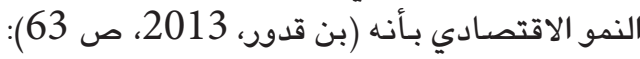
- يجب أن يترتب على الزيادة في الدخل المحلي للبلد زيادة

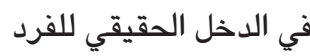

- يجب أن تكون الزيادة في الدخل على المدى الطويل.

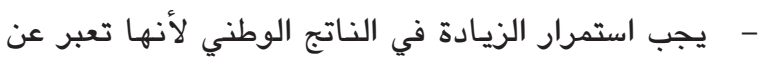

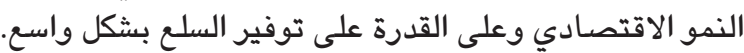

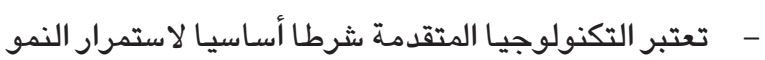

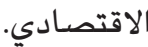

- يستوجب تحقيق النمو المتوقع المصاحب للتكنولوجيا

الجديدة تعديلات مؤسسية وإيديولوجية.

1. العلاقة بين متغيري الدراسة والتعريف بقانون فاجنر:

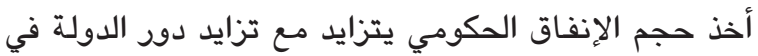

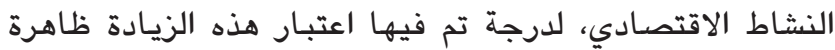

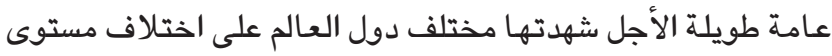

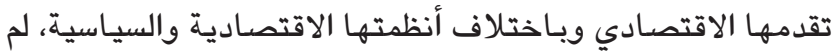

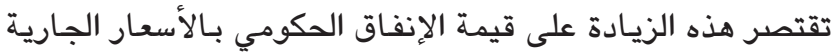

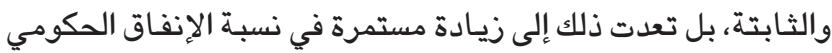

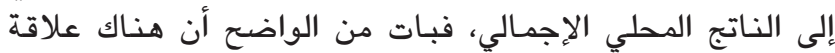

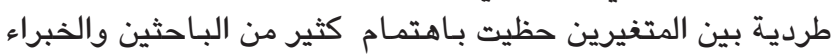

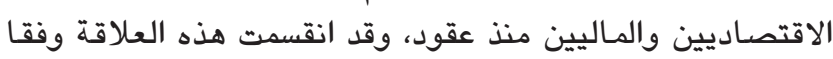

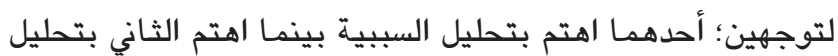

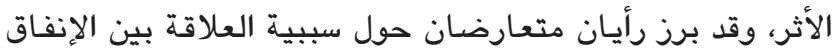

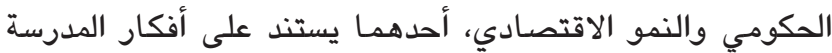

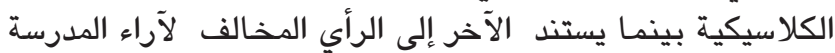

(الكينزية). (الكية بنان.

هذه الدراسة تهتم بالبحث عن سببية العلاقة على ضوء

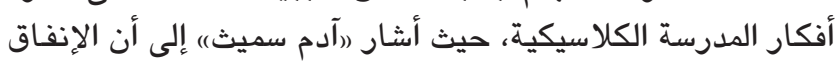

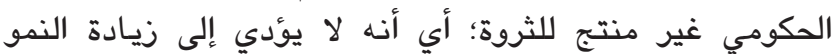

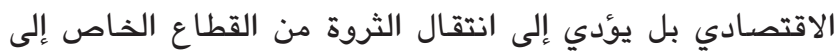

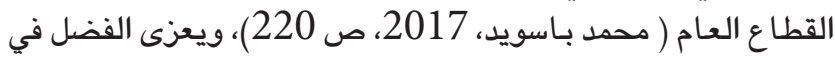

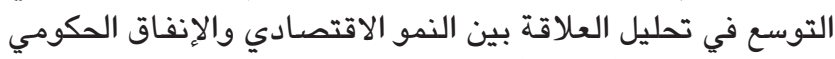

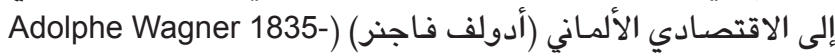

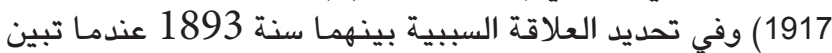

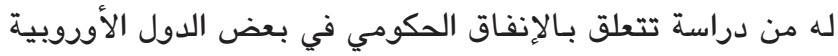

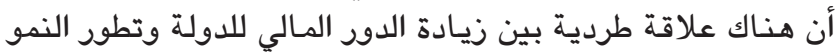

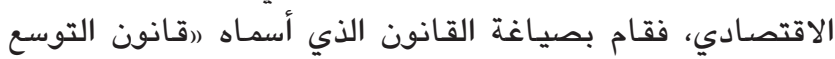

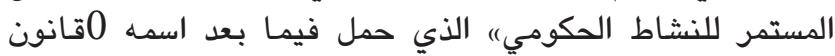

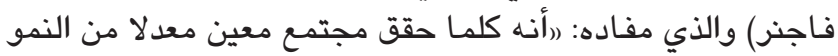

عندما يتحول الاقتصاد من تقليدي إلى صناعي وهو ما يتطابق الجابق

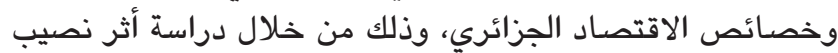

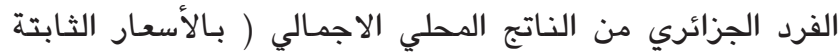

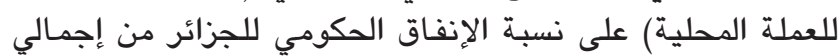

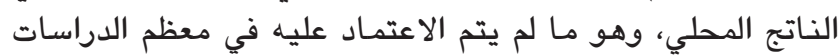

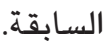

- غطت دراستنا الفترة الزمنية بداية تبني الجزائر لأول

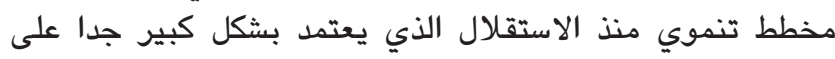

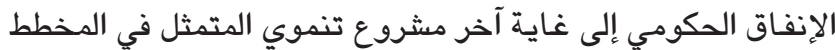

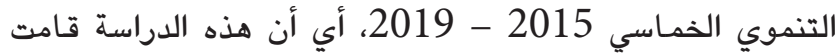
بمسح زمني لكل المشاريع التنموية للجزائر المستقلة.

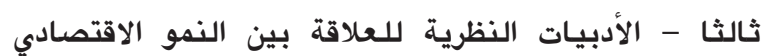

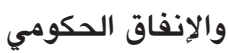

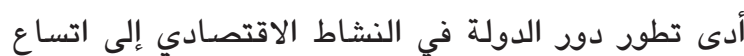

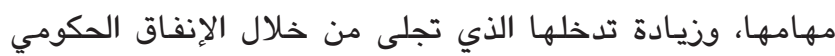

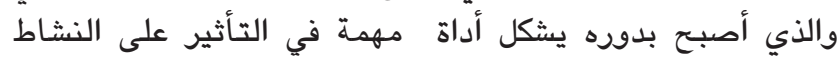

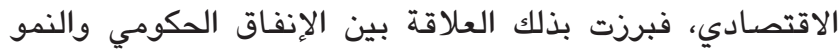

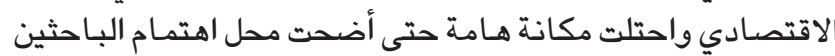

والدارسين الاقتصاديين والماليين منذ قرون إلى يومنا هذا.

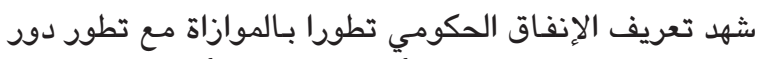

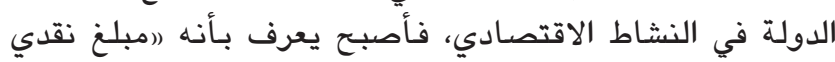

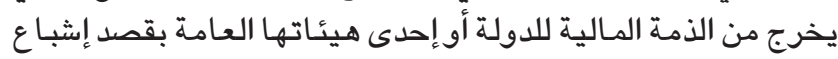

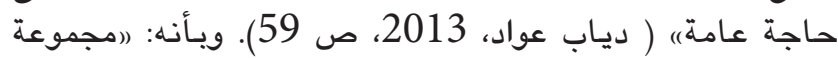
من المصروفات التي تقوم الدولة بإنفاقها في شكل كمية معينة

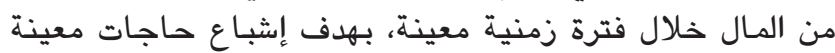

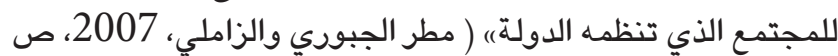

في السابق، وفي ظل الدولة الحارسة لم يكن تقسيم الانفاق

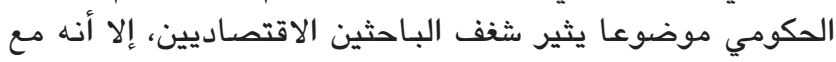

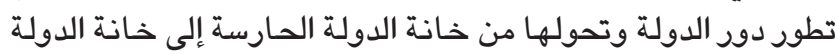

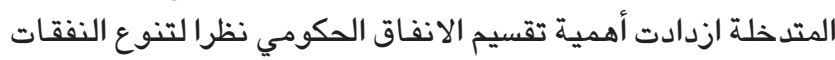

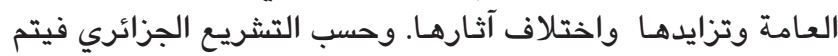

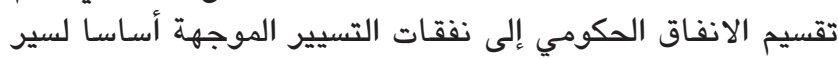

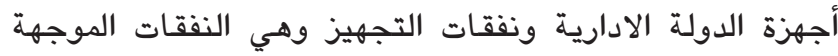

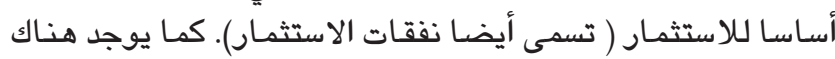

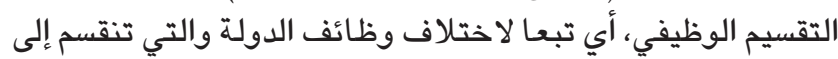

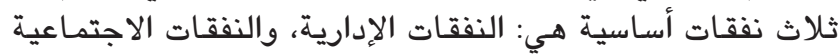

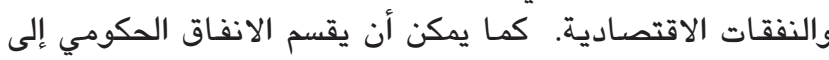

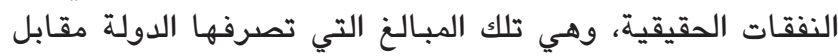

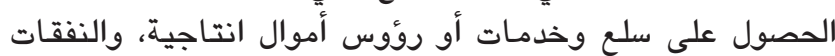

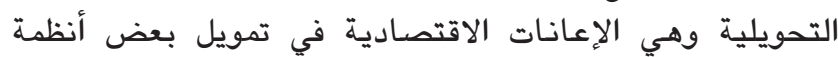

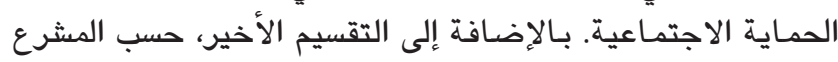

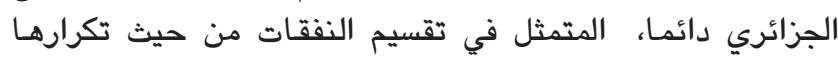

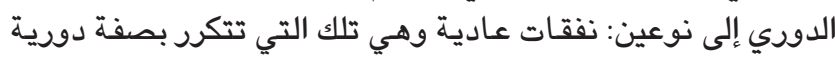

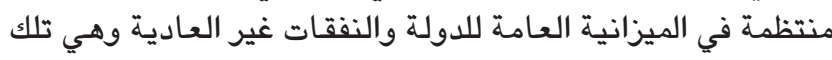

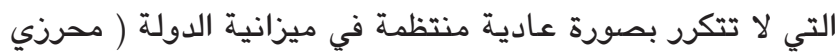
محمد عباس، 2005، ص صن 75 - 92). 
وأبسطها، حيث قدم الباحثان معادلة تعبر عن العلاقة الخطية بين

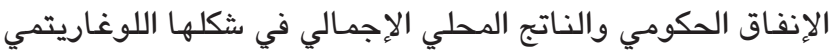

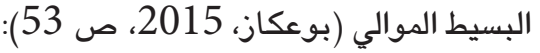

$\operatorname{Ln} G=a+b \operatorname{LnGDP}+u t$

حيث يمثل G الانفاق الحكومي (باللوغاريتم)، و (GDP) الناتج المحلي الاجمالي ( بـاللوغاريتم).

بنى (بيكوك و-ايزمان) طرحهما على أساس أن زيـادة الإنفاق

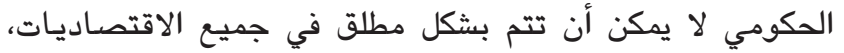

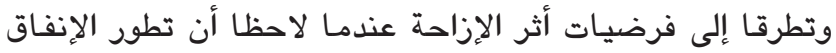

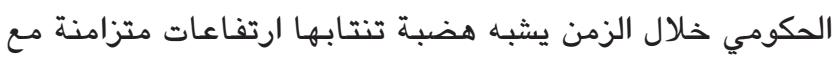

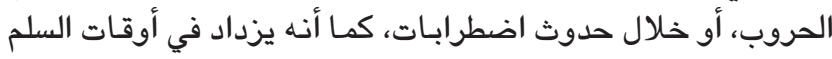

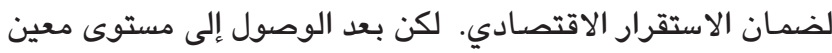

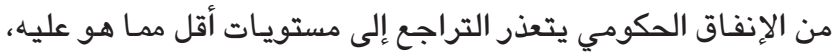

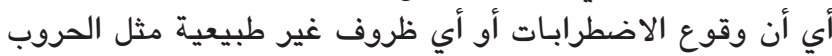

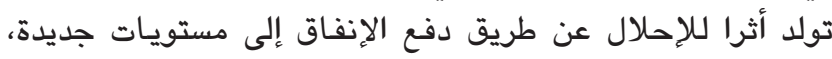

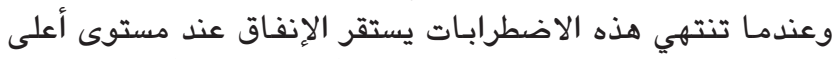

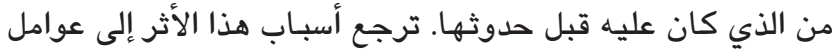

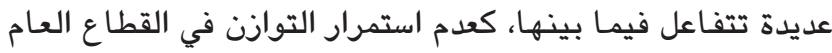
علما أن الإيرادات هي العامل الحاسم في تقرير تلك النفقات. صيغة (كويتا) (1967 Gupta)): اعتبر كويتا المتغير التابع هو

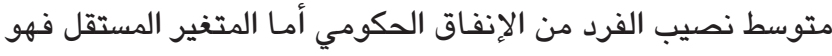

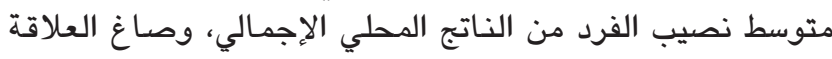
بين النمو الاقتصادي والإنفاق الحكومي في شكلها الوغاريتمي كما الإسا يلي:

$\operatorname{Ln}(G / N)=a+b \operatorname{Ln}(G D P / N)+u t$

حيث يمثل (G): الانفاق الحكومي. (N): عدد السكان. (GDP): الناتج المحلي الاجمالي.

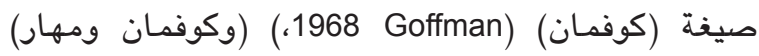

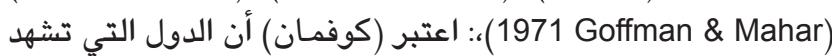

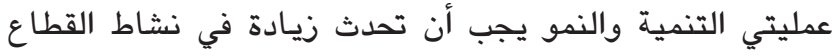

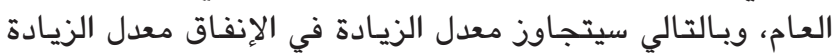

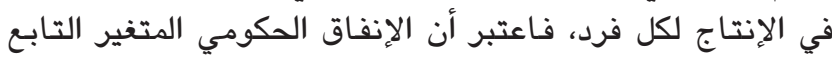

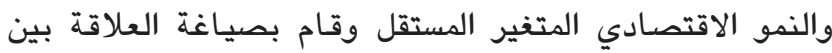

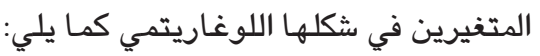

$\operatorname{Ln} \mathrm{G}=\mathrm{a}+\mathrm{b} \operatorname{Ln}(\mathrm{GDP} / \mathrm{N})+\mathrm{ut}$

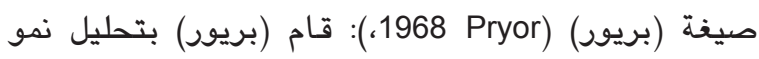

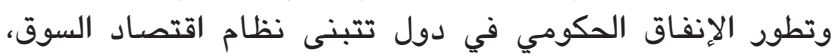

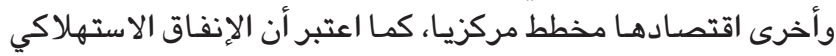

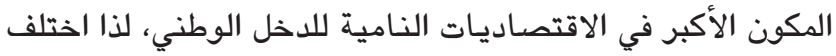

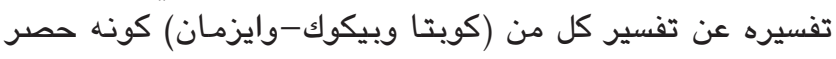

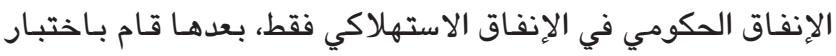

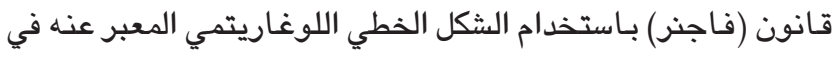

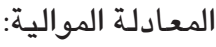

$\operatorname{Ln}(G C / G D P)=a+b \operatorname{Ln}(G D P / N)+u t$

حيث أن المتغير التابع هو نسبة الإنفاق الاستهلاكي على
الاقتصادي يتبعه اتساع في نشاط الدولة المالي، ويـالتالي زيادة

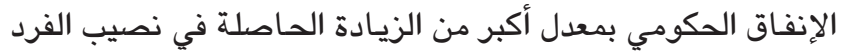

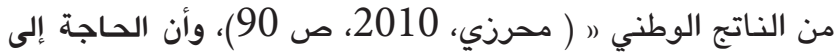
زيادة نفقات الدولـة تعود إلى ثلاثة أسباب:

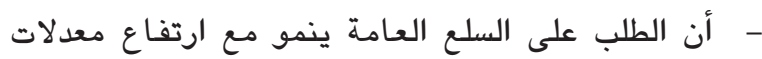

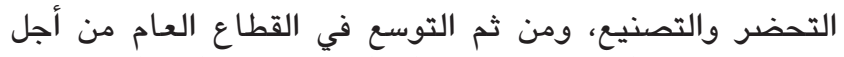

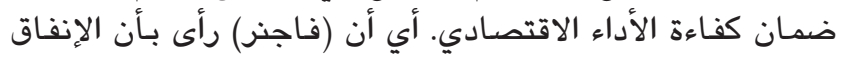

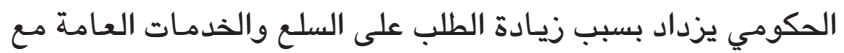

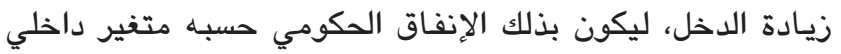

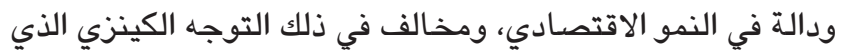

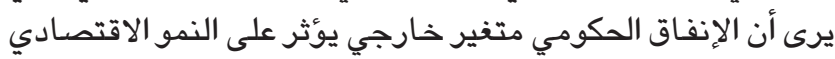

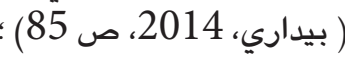

- أن التنمية الاقتصادية تؤدي إلى التوسع في الخدمات

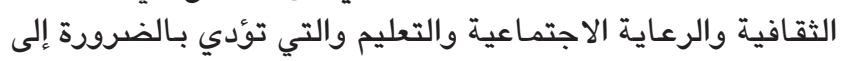
التوسع في الإنفاق الحكومي (Fo- Kossi EdemTogbenu, 2018) ؛ - ـ توفير وتمويل الاستثمارات الضرورية في القطاعات التي

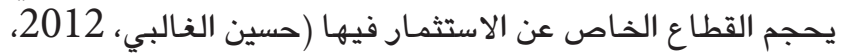

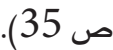

وعليه، يعبر(قانون فاجنر) عن علاقة طويلة الأجل بين

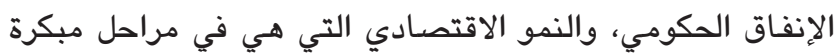

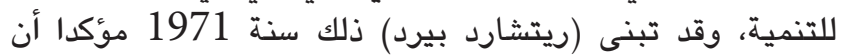

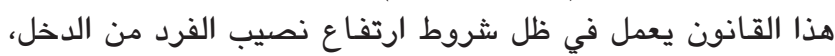

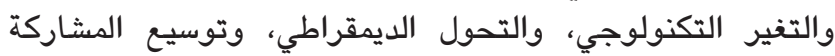

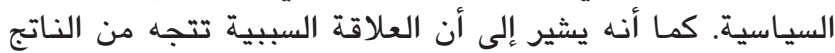

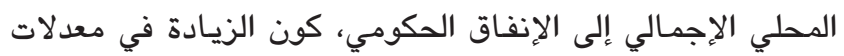

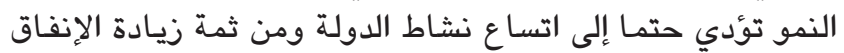

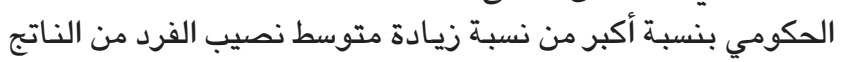

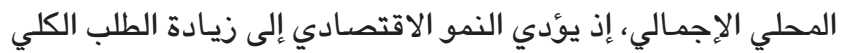

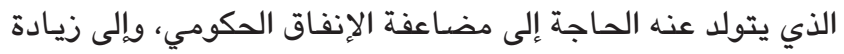

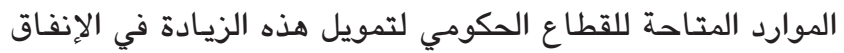

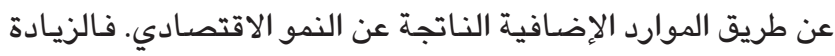

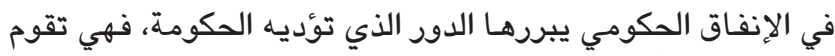

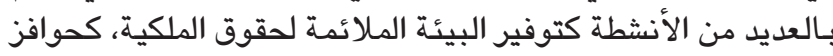

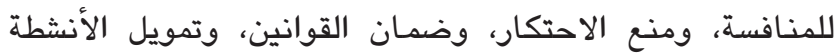

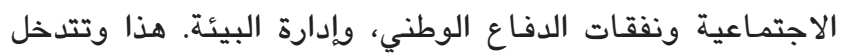

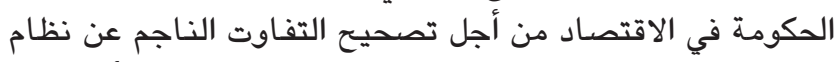
السوق، وللتخفيف من الفقر وإعادة توزيع الدخل والثروة (أبو عيدة،

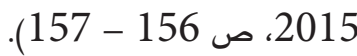

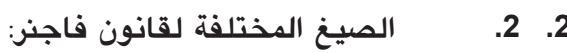

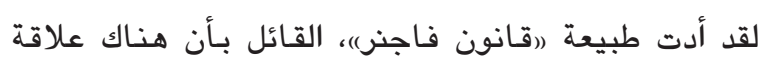

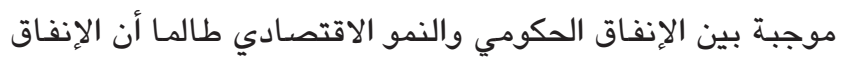

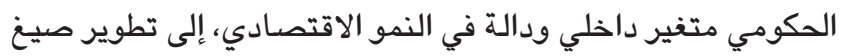

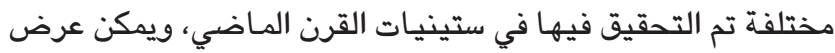
بعض من هذه الصيخ كما يلي:

1961Peacok \& Wi -) سيغة (بيكوك ووايزمان) man 
الموجه 1967 - 1989 شرعت الحكومة الجزائرية في تطبيق أول مخطط ثلاثي سنة

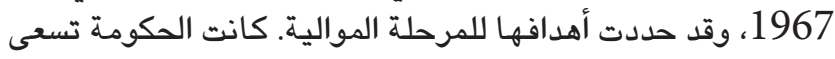

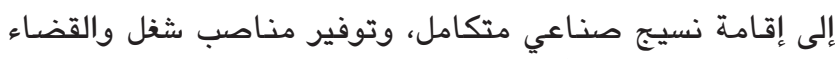

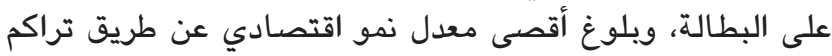

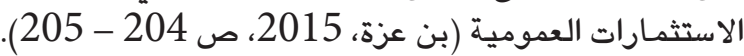

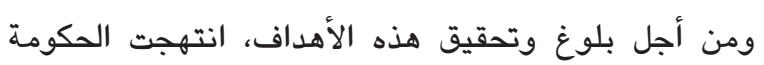

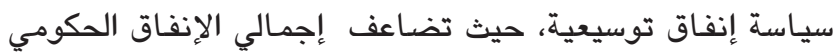

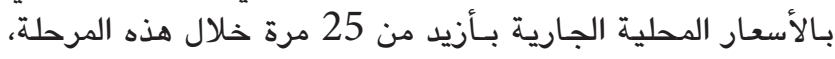

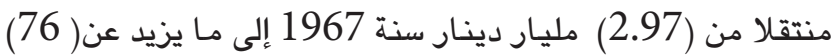

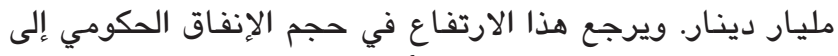

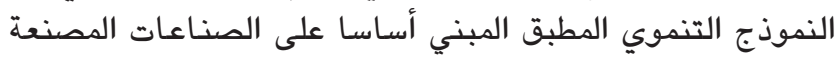

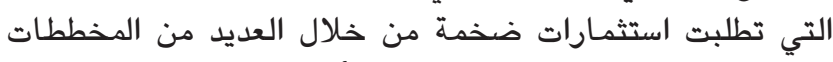

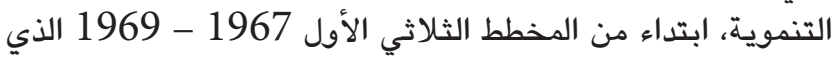
كان مخططا تنمويـا تجريبيا، مرورا بـالمخطط الريـاعي الأول 1973 الأول 1970

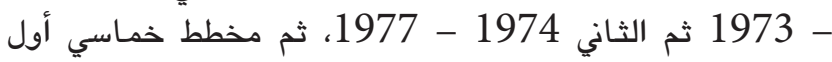

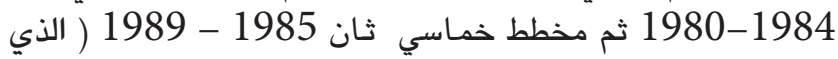
لم يشها تجسيدا على أرض الواقع نتيجة الانهيار المطلق لأسعار

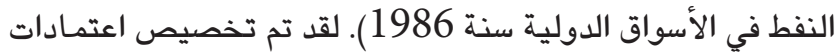

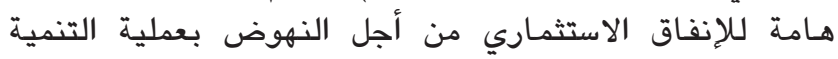

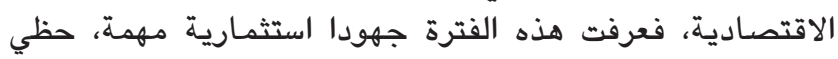

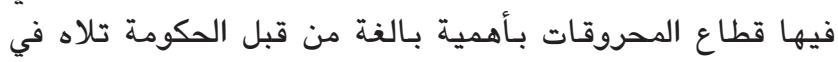

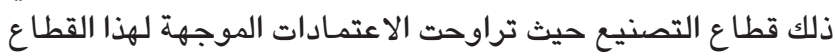

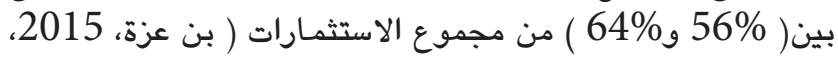

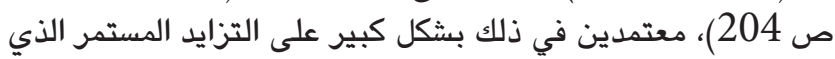

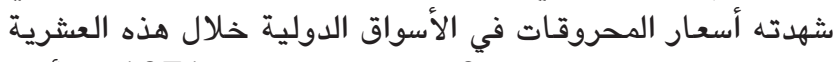

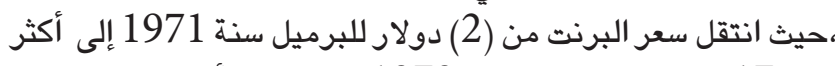

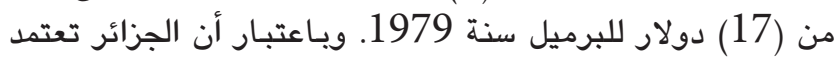
بشكل شبه كلي على المحروقات في صادراتها ( أكثر من 97 \%

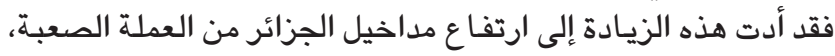

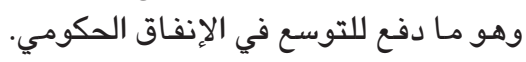

الإنفاق الوطني، والمتغير المستقل هو متوسط نصيب الفرد من

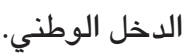
صيغة (ماسغريف) Musgrave، 1969): رأى (ماسغريف)

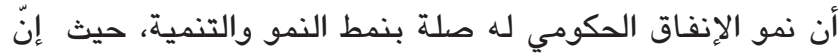

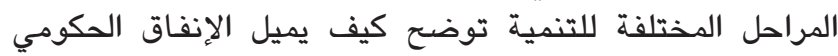

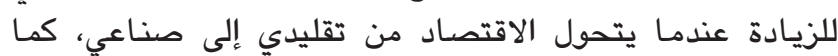

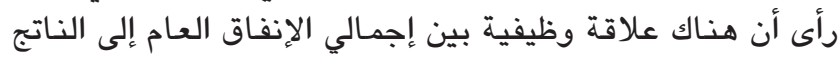

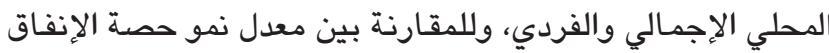

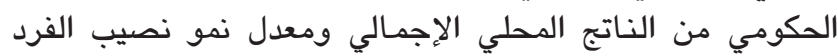

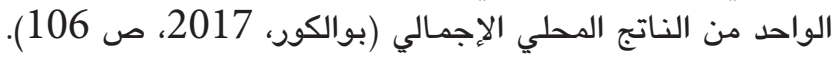
صاغ (ماسغريف) معادلة لاختبار قانون (فاجنر) بـاستخدام الشكل الخطي اللوغاريتمي كما يلي: معادلة لإنيا

$\operatorname{Ln}(G / G D P)=a+b \operatorname{Ln}(G D P / N)+u t$

نموذج (مان) :(Mann, 1980) قام مان بـاختبار جميع تفسيرات وصيخ قانون (فاجنر) السابقة على الاقتصاد المكسيكي

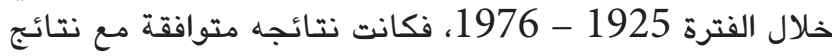

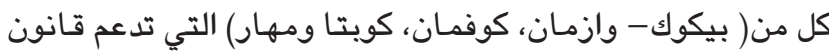

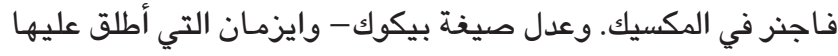

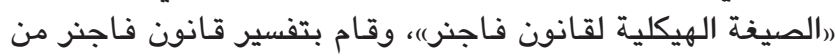

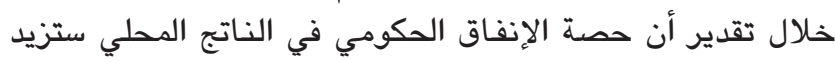

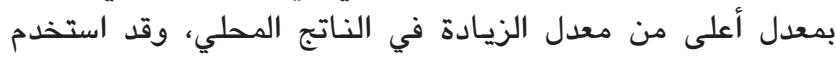
اللوغاريتم الخطي كما توضحه المعادلة الموالية:

$\operatorname{Ln}(G / G D P)=a+b \operatorname{Ln}$ GDP +ut

رابعا - تحليل واقع النمو الاقتصادي والإنفاق الحكومي في الجزائر خلال الفترة : 1967 - 2017

لتتبع تطور كل من الإنفاق الحكومي والنمو الاقتصادي في

الجزائر خلال الفترة 2017-1967، وجب كن الإنغات التمييز بين مرحلتين

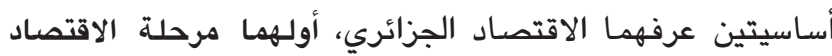

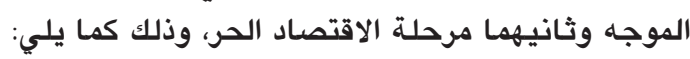
1. الإنفاق الحكومي والنمو الاقتصادي خلال مرحلة الاقتصاد

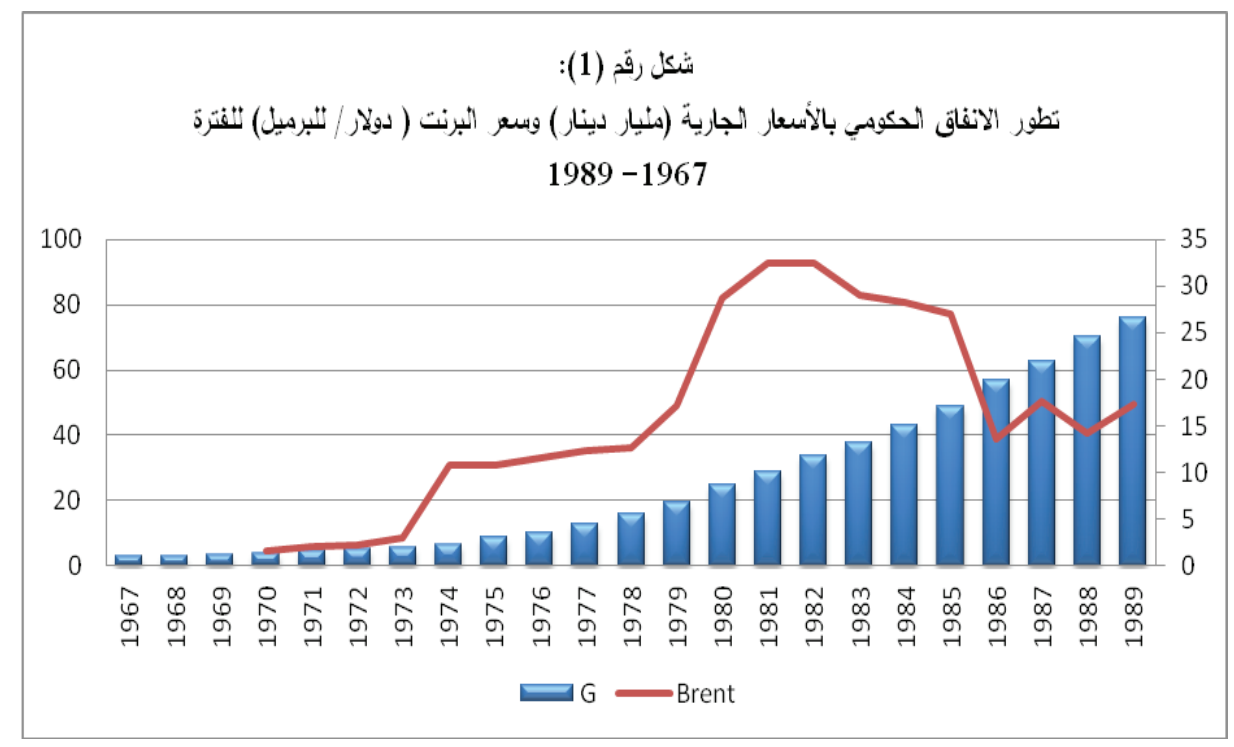

المصدر: من إعداد الباحثين بالاعتماد على احصائيات البنك العالمي 


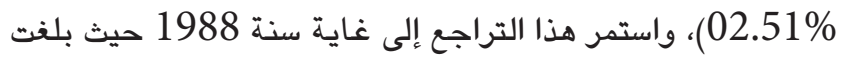

$$
\text { قيمته (108221.78) د دج (انظر الشكل رقم 2). }
$$

بلغت نسبة الإنفاق الحكومي إلى إجمالي الناتج الدحلي

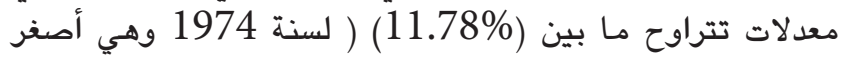

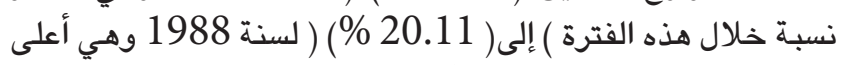
نسبة) كما يظهره الشكل (02) أدناه.
من جهة أخرى أخذ متوسط نصيب الفرد من الناتج المحلي

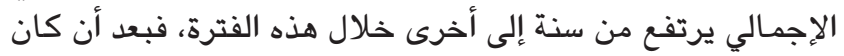

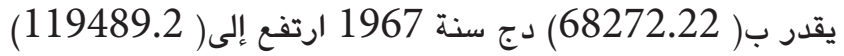

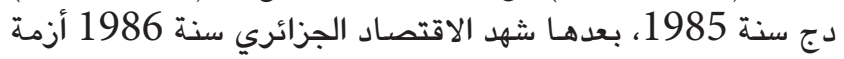
عنيفة بسبب تراجع أسعار النفط في الأسواق الدولية الدائة إلى ألى أدنى

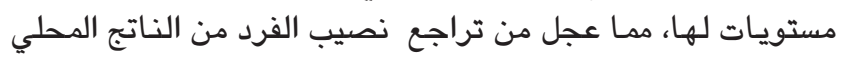

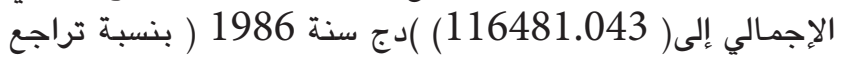

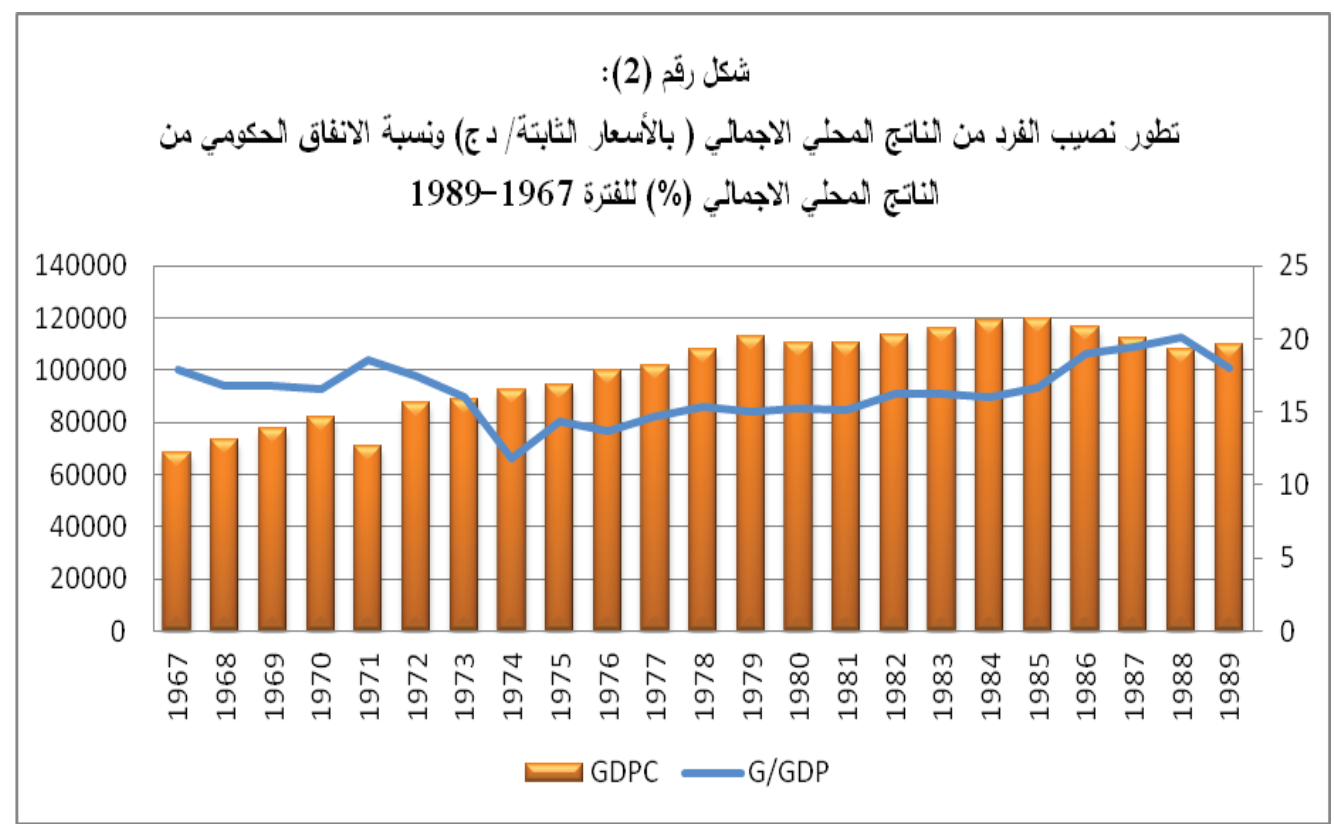

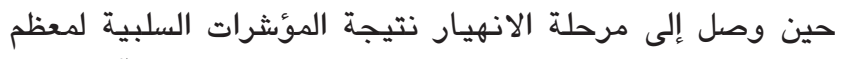

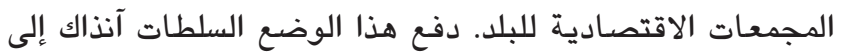

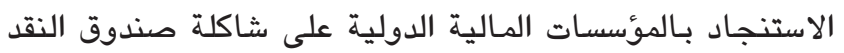

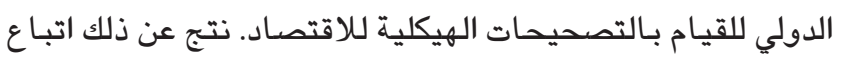

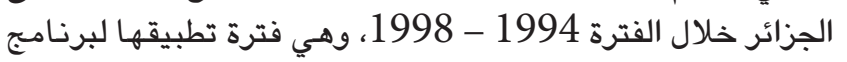

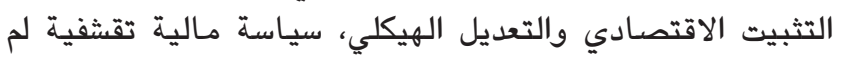

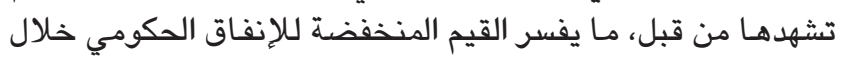

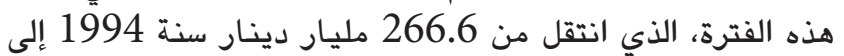

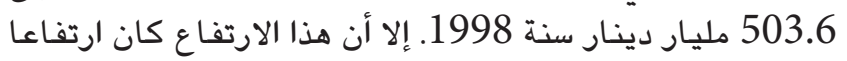

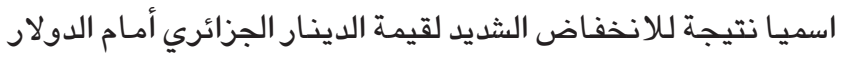

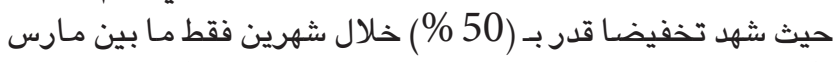
وأفريل 1994 ثم لحقه بعد ذلك تخفيضـات متتالية أدت إلى انتقال

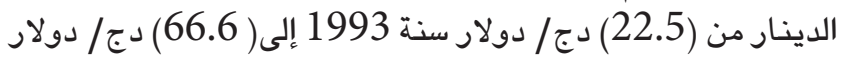

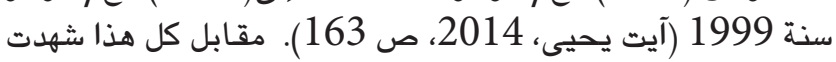
أسعار المحروقات انخفاضـا كبيرا في الأسواق الدولية كانت تتراوح

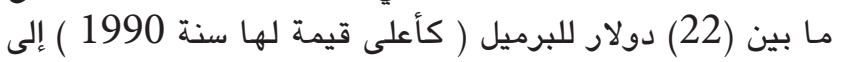

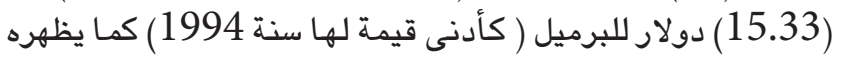

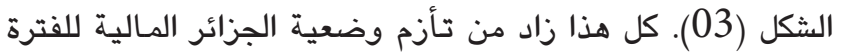

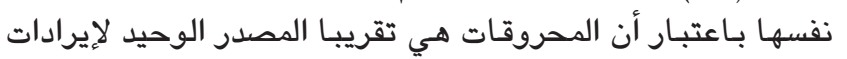

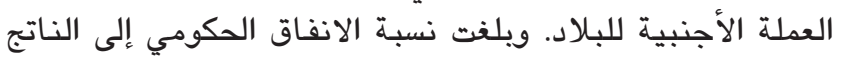

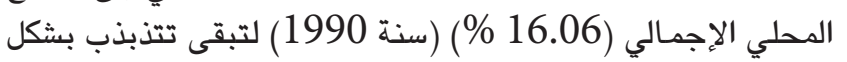
طفيف ومستقرة تقريبا عند هذا المستوى إلى غاية سنة 1999

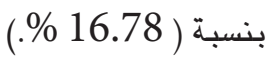

المصدر: من إعداد الباحثين بالاعتماد على احصائيات البنك العالمي

أثبتت الصدمة النفطية لسنة 1986 هشاشة الاقتصاد

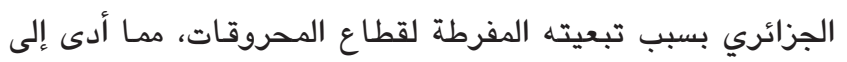

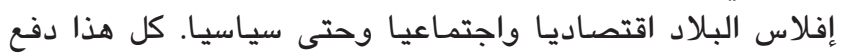

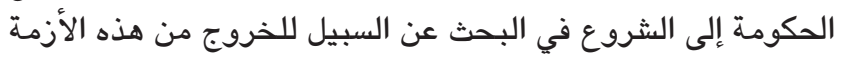

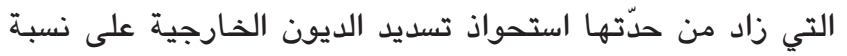

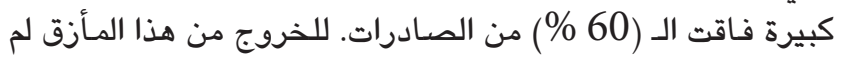

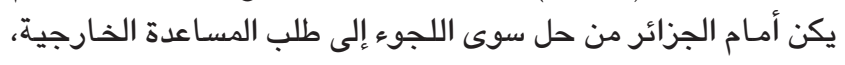

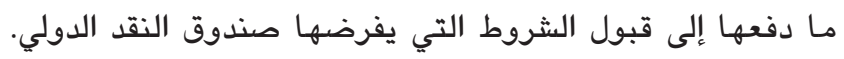

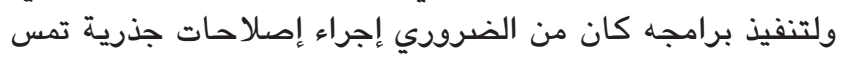

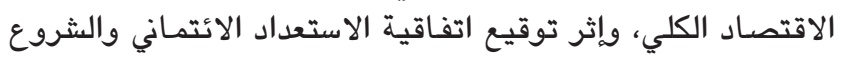

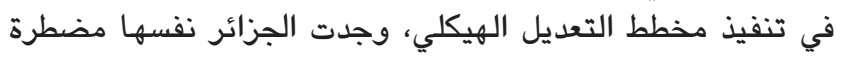

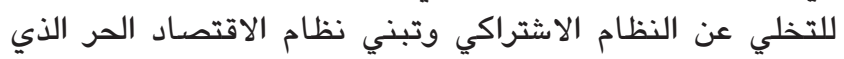

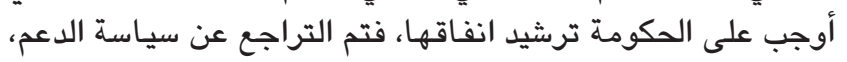

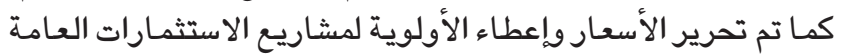

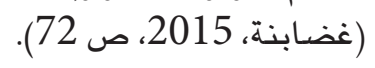

2. الإنفاق الحكومي والنمو الاقتصادي خلال مرحلة الاقتصاد

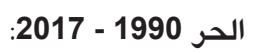

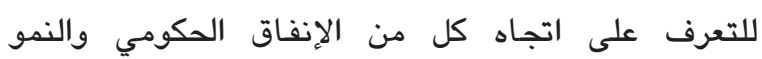
الاقتصادي في الجزائر في ظل هذا النظام الجديد، يمكن التمييز بين الإنين مرحلتين هما:

1 -2 مرحلة الاصلاحات الهيكلية 1990 - 1998: تعتبر سنوات 1990 من أصعب سنوات الاقتصاد الوطني، 


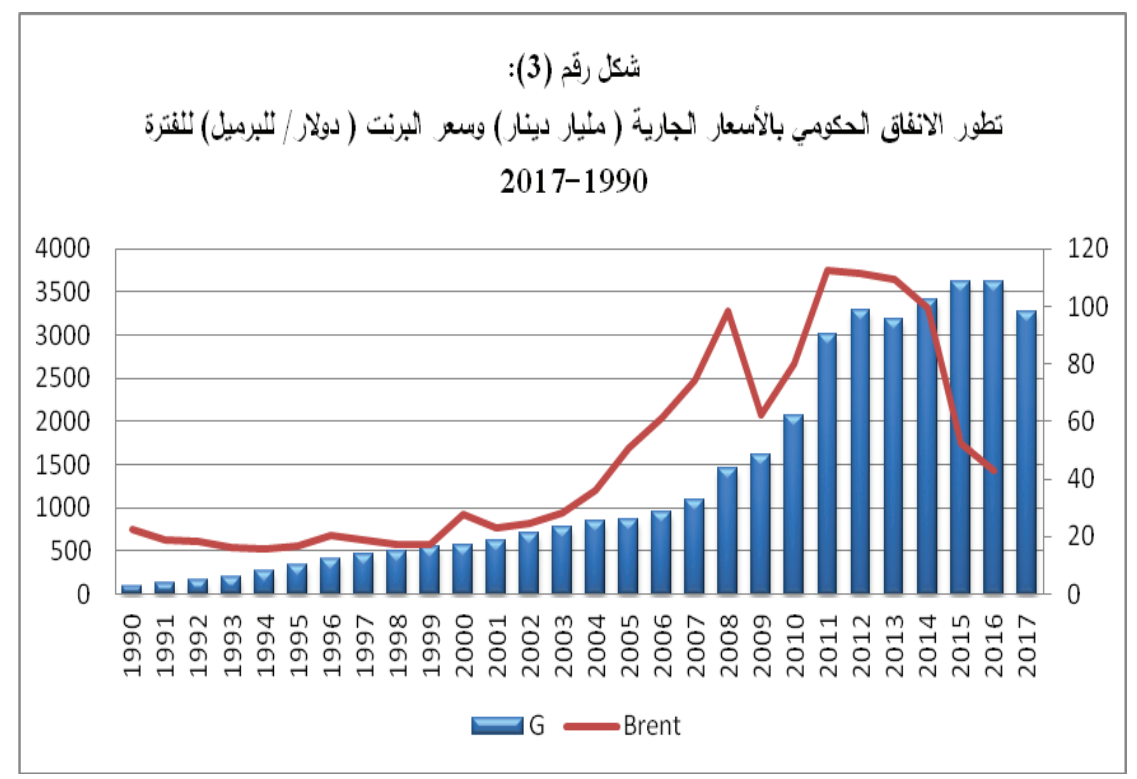

المصدر : من إعداد الباحثين بالاعتماد على احصائيات البنك العالمي

من سنة 1994 إلى 1998 يمكن القول إنّ نصيب الفرد من الناتج

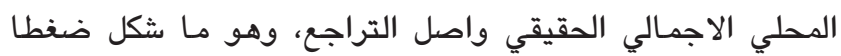

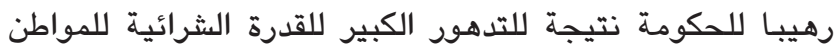

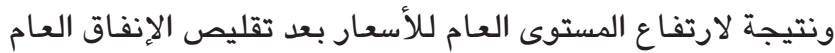

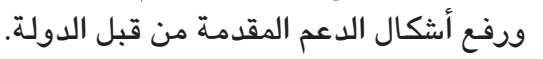

في المقابل كان نصيب الفرد من الناتج المحلي الإجمالي

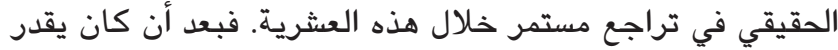

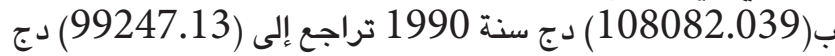

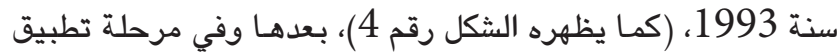

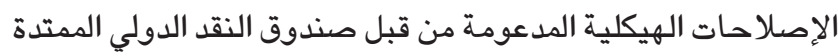

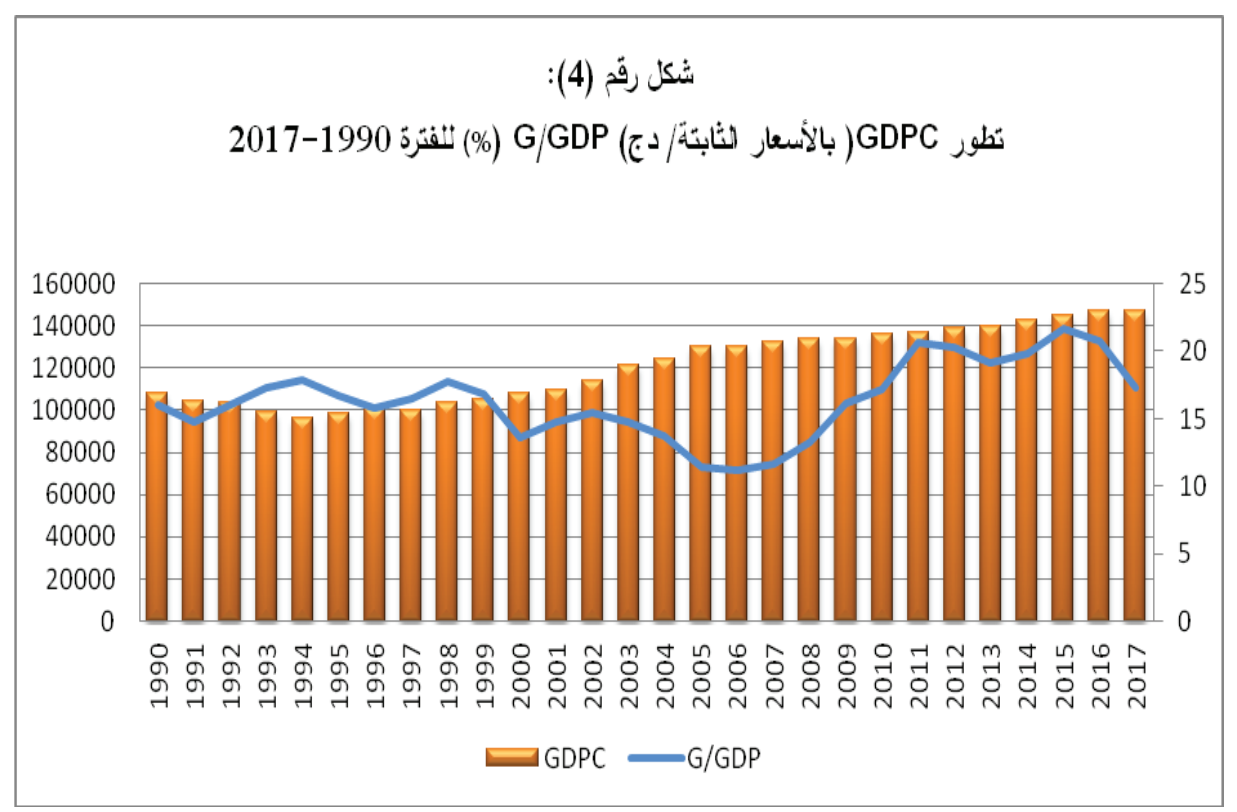

المصدر: من إعداد الباحثين بـالاعتمـاد على احصائيـات البنك العالمي

الحكومي، عبر برامج الاستثمارات التي تم إنجازهـا خلال الفترة

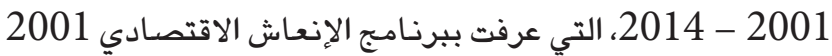

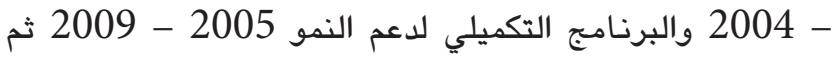

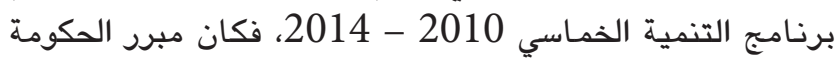
لانتهاج هذه السياسة ضرورة العمل على تدارك التأخر في التنمية التمدية الموروث عن الأزمة المتعددة الأبعاد „الاقتصادية، والسياسية،
2 - 2 مرحلة الإنعاش الاقتصادي 1999 - 2017 شهدت أسعار النفط في الأسواق الدولية نهاية سنة 1999

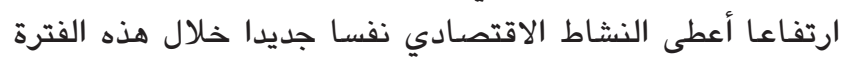

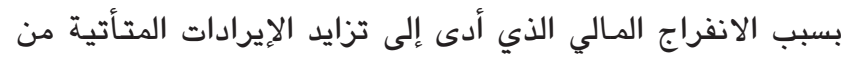

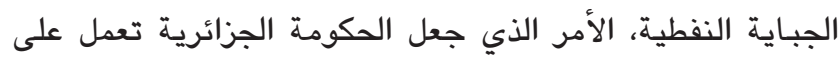
انتهاج سياسة مالية توسيعية، من خلال التوسع في الإنفاق التئ تعلئ 
سنة 1967 (وهي أول سنة شرعت فيها الحكومة الجزائرية بتطبيق أول مخطط ثلاثي إلى غاية سنة 1967 (وهي ألى 2017 وهي آخر سنة تتوفر عنها

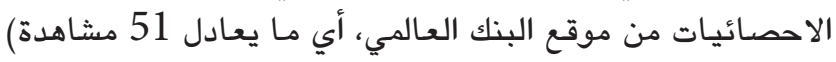

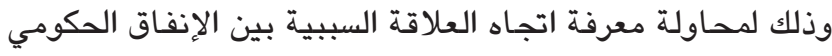

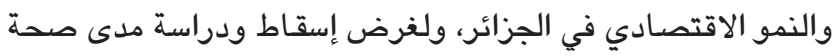

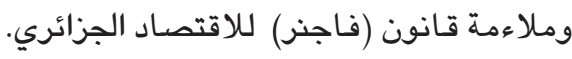

لكي لا يتم اختبار الصيغ الست المفسرة لقانون

(فاجنر) والتي تم ذكرهـا آنفا، سيتم هنا الاعتمـاد على نموذج

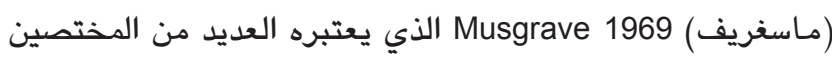
الصيغة الأكثر ملاءمة لاختبار قانون (فاجنر)، ومن الدراسات

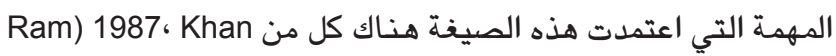
و Murthy الصيغة التالية: - n

$\frac{d g}{d g d p c}>0 \frac{G}{G D P}=f\left(\frac{G D P}{N}\right)$ g $g=f(g d p c)$

حيث أن و: هو نسبة الانفاق الحكومي للجزائر (G) من إجمالي

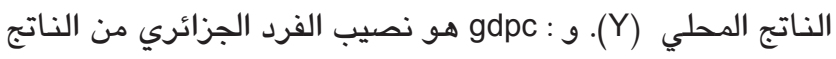

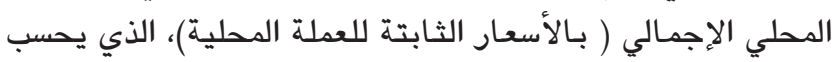

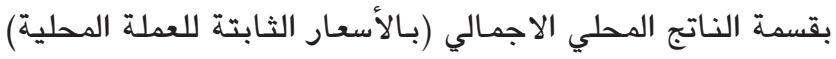
(GDP) على المتفيرين لتصبح المعادلة من الشكل:

$\ln g_{t}=\alpha_{0}+\alpha_{1} \operatorname{lngdpc_{\mathrm {t}}}+v_{t}$

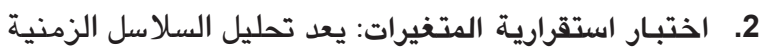

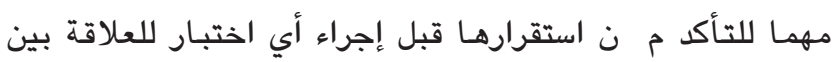

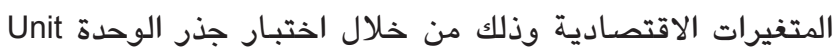

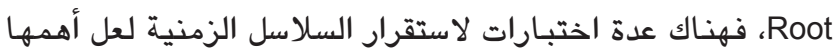

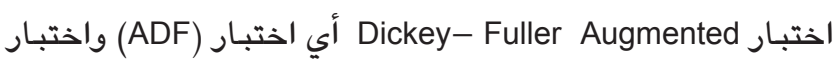
(PP) ،وهما ما سيتم اختبارهما (Phillips -Perron على متغيرات هذه الدراسة.
والمالية، والاجتماعية والأمنية، التي عاشتها البلاد خلال الفترة

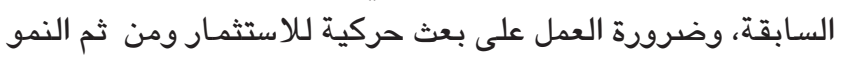

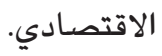

خلال الفترة التي شُرع فيها في تنفيذ هذه البرامج التنموية

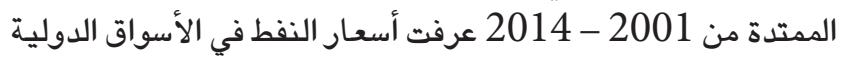

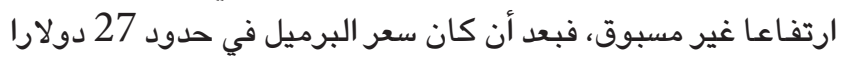

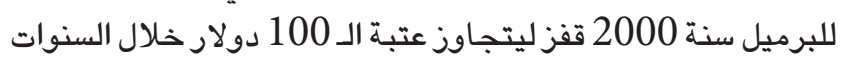

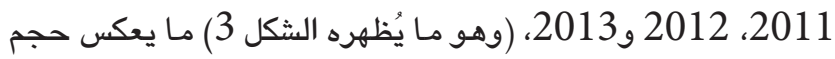

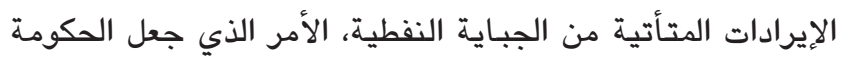

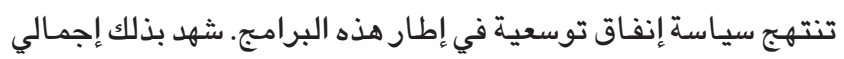

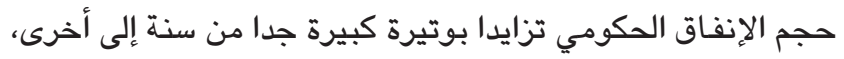

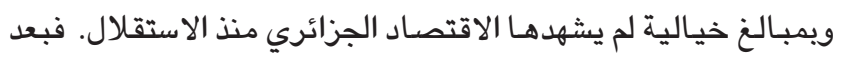

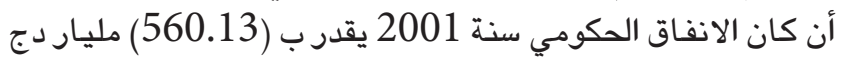
ارتفع إلى 2017 الانياف 3617 مليار دج سنة 2016، لينخفض بشكل طفيف

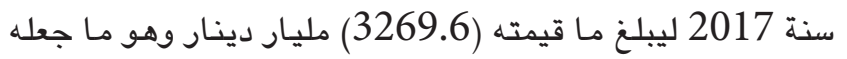

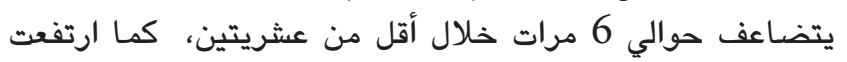
نسبته إلى إجمالي الناتج المحلي من (14.77 ماتل

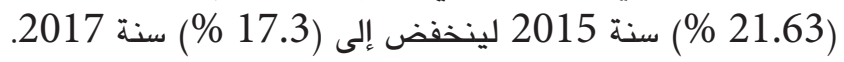

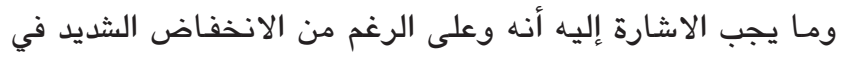

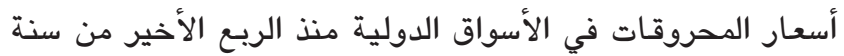

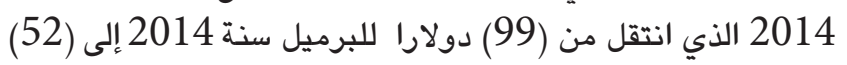

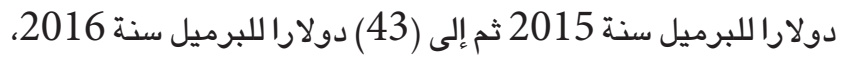

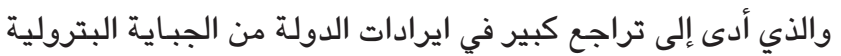

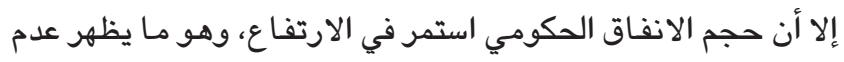

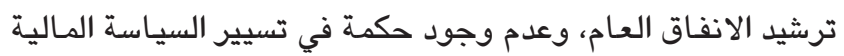

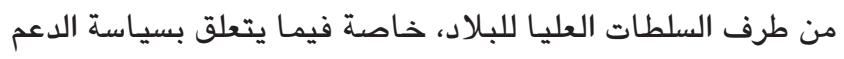

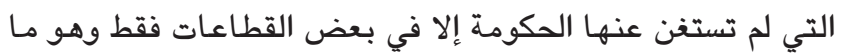

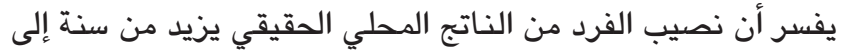

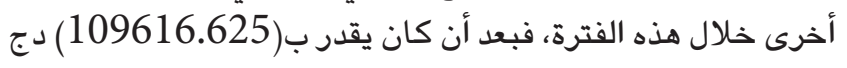

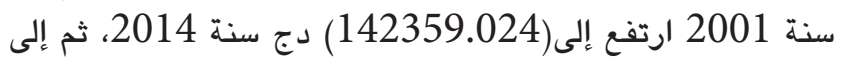
(146904.3) مليون دج سنة إلى 2017.

خامسا- الدراسة القياسية لعلاقة النمو الاقتصادي بـالإنفاق

الحكومي في الجزائر خلال الفترة: 1967 - 2017 1. توصيف بيانات ومتغيرات الدراسة:

تم الاعتماد على سلسلة من البيانات السنوية، التي امتدت من لن

جدول (1): - 2 (1)

اختبارات الجذر الوحدوي لمتغيرات الدراسة حسب ADF و جP

\begin{tabular}{|c|c|c|c|c|c|c|}
\hline \multirow[b]{2}{*}{ النتيجة } & \multicolumn{2}{|c|}{ عند الفارق الأول } & \multicolumn{2}{|c|}{ عند المستوى } & \multirow[b]{2}{*}{ المتغير } & \\
\hline & t-PP & $\mathrm{t}-\mathrm{ADF}$ & $\mathrm{t}-\mathrm{PP}$ & $\mathrm{t}-\mathrm{ADF}$ & & \\
\hline (I 1) & $* * *-2.922449$ & $* * *-2.922449$ & -2.921175 & -2.922449 & الثابت & \\
\hline (I 1) & $* * *-3.504330$ & $* * *-3.504330$ & -3.502373 & -3.504330 & الاتجاه العام والثابت & Lng \\
\hline
\end{tabular}




\begin{tabular}{|c|c|c|c|c|c|c|}
\hline \multirow[b]{2}{*}{ النتيجة } & \multicolumn{2}{|c|}{ عند الفارق الأول } & \multicolumn{2}{|c|}{ عند المستوى } & \multirow{2}{*}{\multicolumn{2}{|c|}{ المتغير }} \\
\hline & $\mathrm{t}-\mathrm{PP}$ & $\mathrm{t}-\mathrm{ADF}$ & $\mathrm{t}-\mathrm{PP}$ & $\mathrm{t}-\mathrm{ADF}$ & & \\
\hline (I 1) & $* * *-2.922449$ & $* * *-2.922449$ & -2.921175 & -2.922449 & الثابت & \\
\hline (I 1) & $* * *-3.504330$ & $* * *-3.504330$ & -3.502373 & -3.518090 & الاتجاه العام والثابت & Lngdpc \\
\hline
\end{tabular}

*، ، * *** تعني رفض فرضية العدم ( استقر ار السلسلة) عند مستويات 10\%، 5 \% و 1\% على الترتيب.

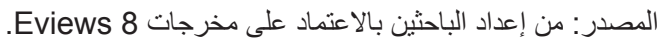

مستقرين عند المستوى، ولكنهما مستقران عند الفارق الأول سواء

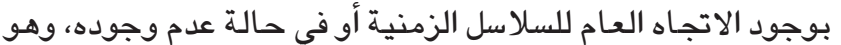

ما يؤكده الشكل البياني (05).
تُظهر نتائج اختبار كل من( ديكي فولر) الموسع واختبار

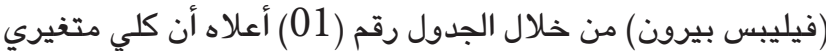

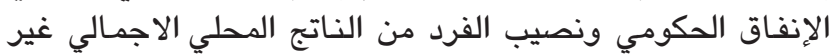

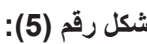

$$
\text { تطور متغيرات البحث حسب الزمن }
$$

LNGDPC

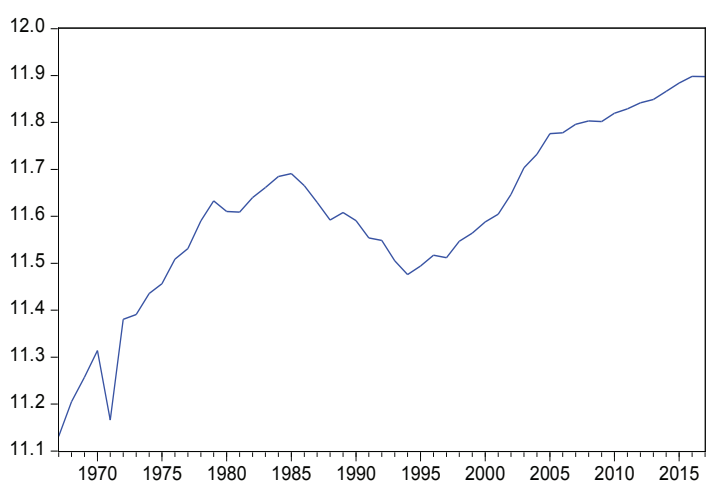

DLNGDPC

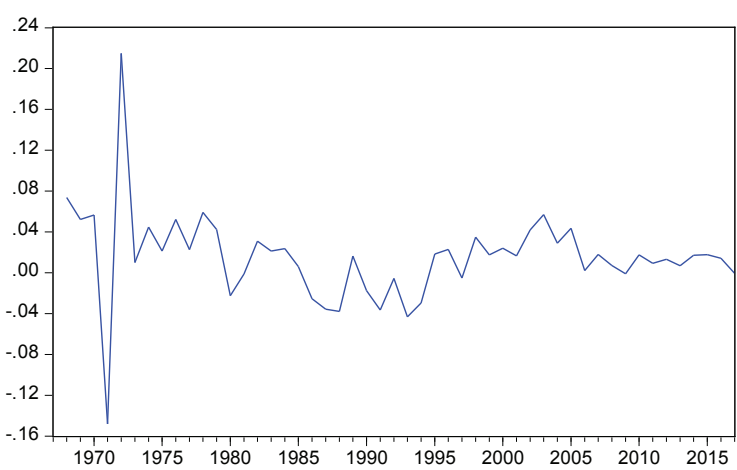

عدد متجهات التكامل المشترك الفريدة يقل عن أو يساوي العدد (q) عالئل الفرض البديل (r=q) ويحسب بـالصيغة التالية:

$$
\lambda_{\text {Trace }}(r)=-T \sum_{i=r+1}^{p} \ln \left(1-\lambda_{i}^{*}\right)
$$

بحيث تمثل (T):) حجم العينة ، (r): عدد متجهات التكامل

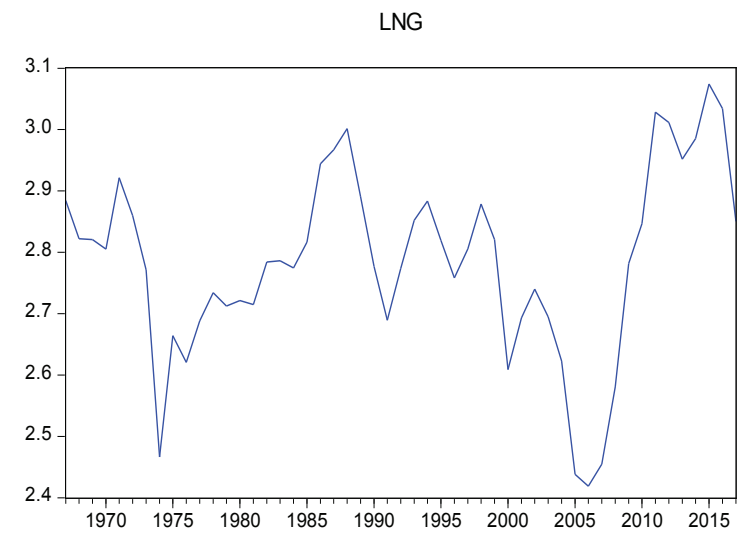

DLNG

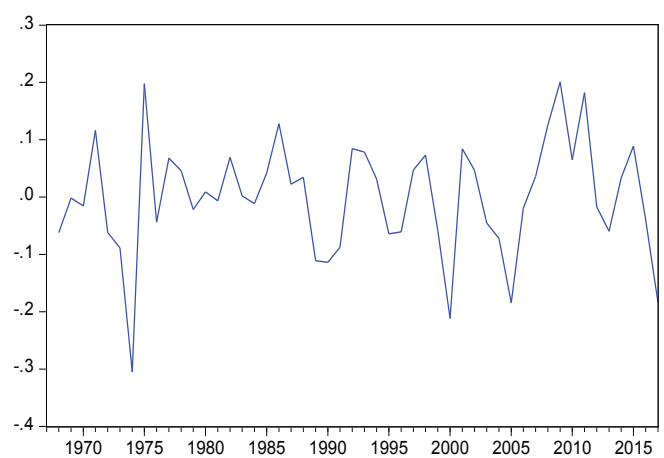

Eviews 8 المصدر: من مخرجات

3. اختبار التكامل المشترك Co-integration:

إن تحليل التكامل المشترك الذي يسمى انحدار التكامل

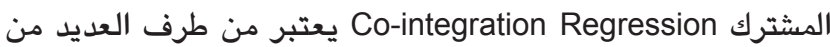

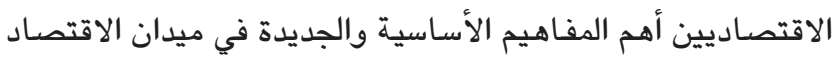
القياسي وتحليل السلاسل الزمنية وسيتم خلال هذه الدراسة اختبار الاسيار

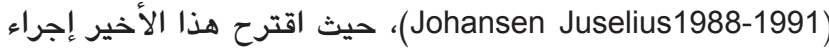
اختبارين لتحديد عدد متجهات التكامل المشترك: * اختبـار الأثر (Trace) : يتم اختبـار فرضية العدم القائلة بـأن المشترك. 
على وجود (r+1) من متجهات التكامل المشترك. فإذا زادت القيمة

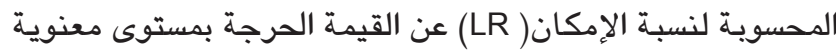

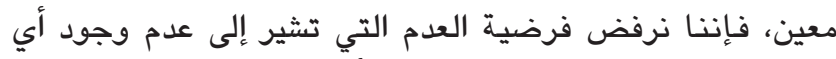

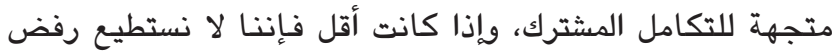

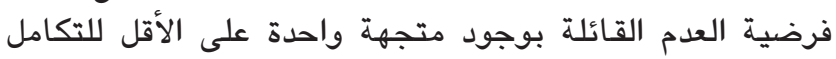
المشترك. والجدول (02) يوضح المتح اختبار (جوهانسن) للتكامل المشترك عند درجة معنوية( 5 \%).
× اختبار القيمة القصوى (Max $\lambda$ (اقلية): الذي تحسب إحصائيته

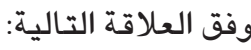

$$
\lambda_{\text {Max }}(r, r+1)=-T \ln \left(1-\lambda_{r-1}^{*}\right)
$$

ويجرى اختبار فرضية العدم التي تنص على وجود (r) من متجهات التكامل المشترك مقابل الفرضية البديلة التي تنص ونص

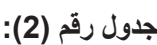

اختبار التكامل المشترك حسب جو هانسن لمتنيرات الدراسة

\begin{tabular}{ccccc}
\hline \multicolumn{4}{c}{ Trace 1 Trace } & 0.05 \\
\hline Hypothesized & & Statistic & Critical Value & Prob.** \\
No. of CE(s) & Eigenvalue & 17.36998 & 15.49471 & 0.0258 \\
None * & 0.265961 & 2.528711 & 3.841466 & 0.1118 \\
At most 1 & 0.051318 & & \\
\hline
\end{tabular}

\section{Maximum Eigenvalue اختبار}

Hypothesized

Max-Eigen

0.05

No. of CE(s)

Eigenvalue

Statistic

Critical Value

Prob.**

None *

0.265961

14.84127

14.26460

0.0405

At most 1

0.051318

2.528711

3.841466

0.1118

المصدر: من إعداد الباحثين بالاعتماد على مخرجات EViews 8

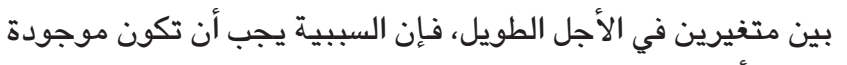

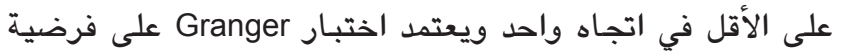

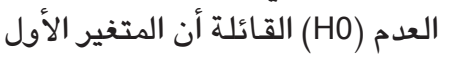

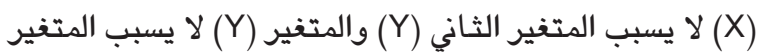

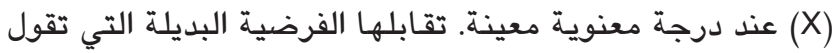

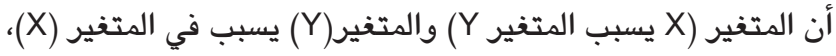

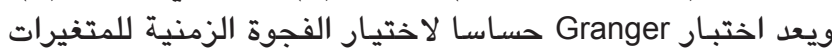

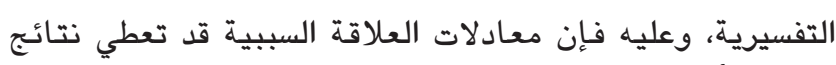

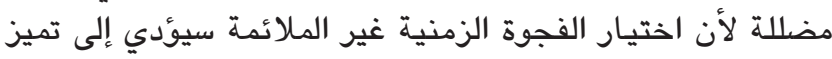
النتائج. ومن أجل التغلب على المشاكل المصاحبة لعدم اختيار الفجوة

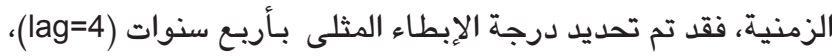
وذلك حسب اختبار كل من ( AIC): Akaike information criterion و رابتخاو HPE: Final prediction error Hannan-Quinn inبالإضافة إلى اختبار (LR) formation criterion

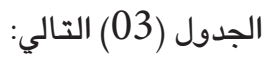

يُظهر الجدول (02) أعلاه، وجود تكامل مشترك واحد على إلى الأقل بين متغيري الدراسة (r=1) سواء باء بالنسبة لاختبار

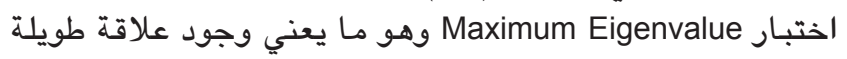

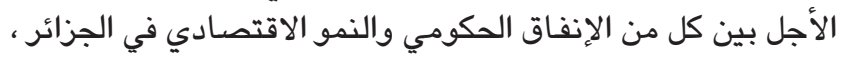

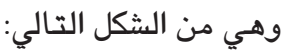
Ln $g=9.06+1.018$ Ln gdpc

هذه المعادلة تدل على وجود علاقة طردية بين كل من

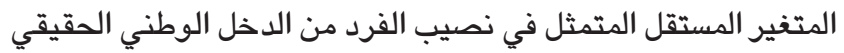

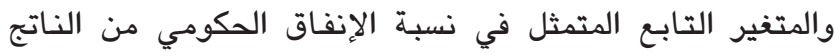

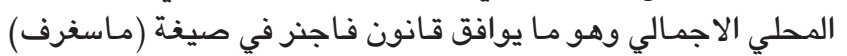

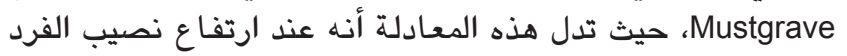

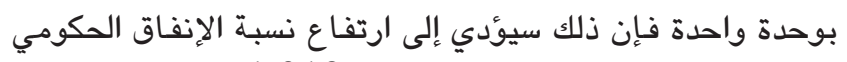

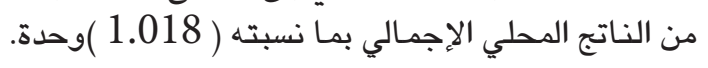

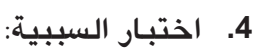

تعد طريقة Granger أكثر الطرق المستخدمة لاختبار

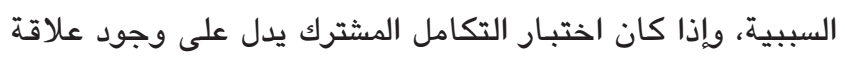

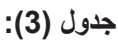

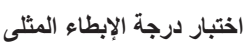

\begin{tabular}{ccccccc}
\hline Lag & LogL & LR & FPE & AIC & SC & HQ \\
\hline 0 & 41.60907 & NA & 0.000635 & -1.685492 & -1.606763 & -1.655866
\end{tabular}


مجلة جامعة القدس المفتوحة للبحـوث الإدارية والاقتصادية - الجملد (4) - ع (12) - كانون الأول 2019

\begin{tabular}{ccccccc}
\hline Lag & LogL & LR & FPE & AIC & SC & HQ \\
\hline 1 & 125.1674 & 156.4497 & $2.15 \mathrm{e}-05$ & -5.070954 & $-4.834765^{*}$ & -4.982074 \\
2 & 130.6083 & 9.724130 & $2.03 \mathrm{e}-05$ & -5.132268 & -4.738619 & -4.984135 \\
3 & 135.0195 & 7.508492 & $2.00 \mathrm{e}-05$ & -5.149767 & -4.598659 & -4.942382 \\
4 & 142.2123 & $11.63092 *$ & $1.75 \mathrm{e}-05^{*}$ & $-5.285631 *$ & -4.577064 & $-5.018993 *$ \\
\hline
\end{tabular}

Eviews 8 المصدر: من إعداد الباحثين بالاعتماد على مخرجات

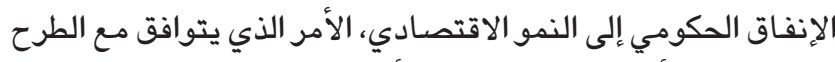

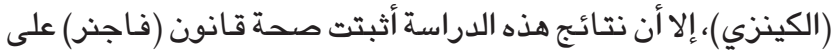

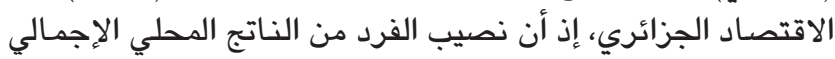

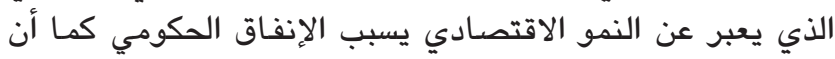

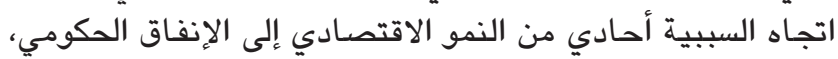

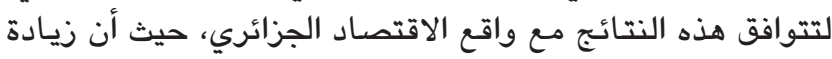

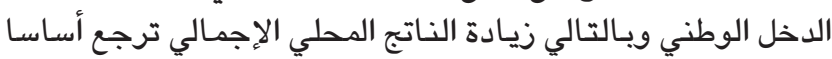

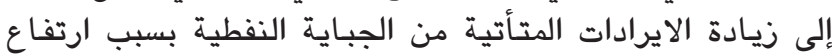

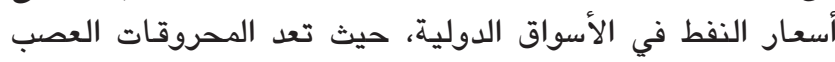

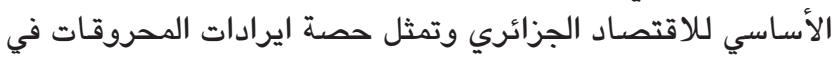

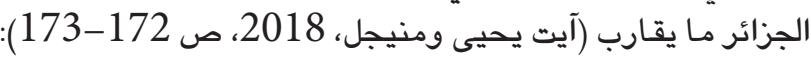

$$
\begin{aligned}
& \text { (66 \% \% م \% من الناتج المحلي الإجمالي. } \\
& \text { (66 \% \% من الايرادات العامة لميزانية الدولة. } \\
& \text { ( } 98 \text { \% من مجموع عائدات التصدير. }
\end{aligned}
$$

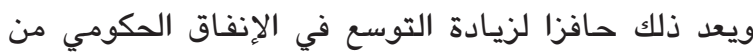

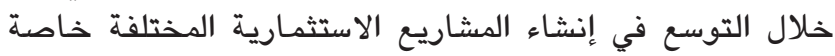

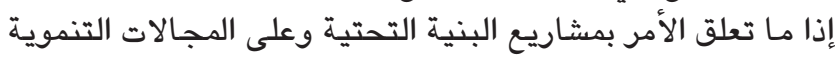

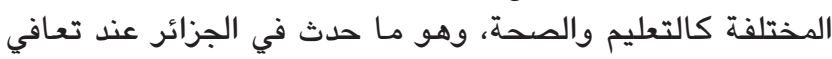

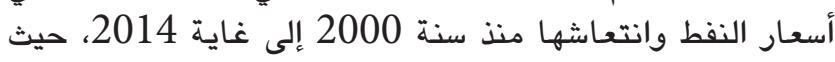

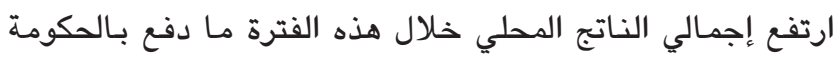

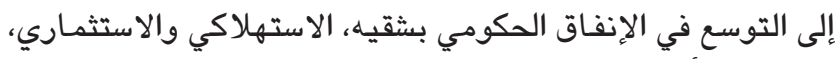

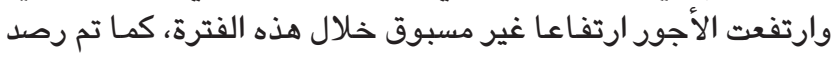

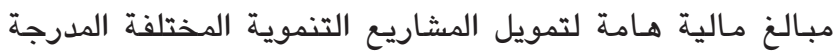
ضمن برامج الإنعاش الاقتصادي.

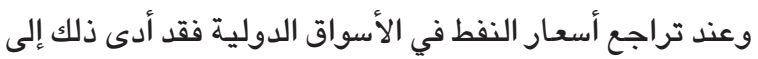

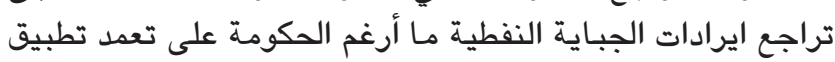

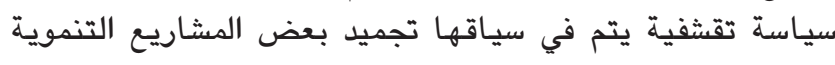

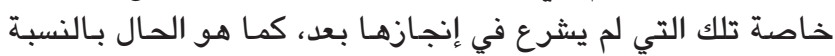

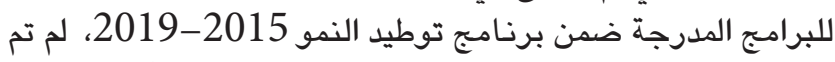
تجميد البرامج التي لم يشرع في إنجازهـا عقب استمرار أسعار النفط

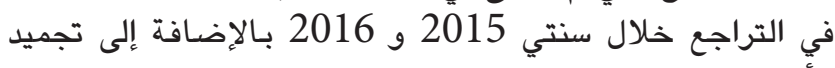

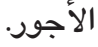

\section{سادساـ الثتائج والتوصيات:}

تم التوصل من خلال هذه الدراسة إلى النتائج التالية:

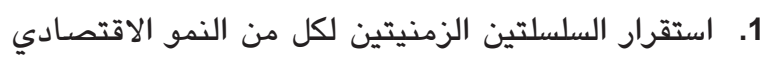

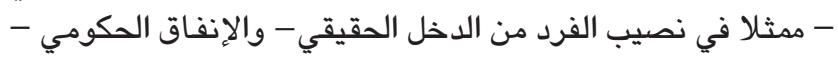

كما يبين الجدول (04) العلاقة السببية بين متغيري الدراسة.

\begin{tabular}{|c|c|c|c|}
\hline Prob & F-Statistic & الملاحظات & الفرضية الصفرية \\
\hline 0.0045 & 4.51709 & & (D Lng) لا يسبب (D Lngdpc) \\
\hline 0.9758 & 0.11659 & 47 & لا يسبب (D Lng) \\
\hline
\end{tabular}

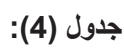

Granger اختبار سبية كرانجر (4):

Eviews 8 المصدر: من إعداد الباحثين بالاعتماد على مخرجات برنامج

يمكن تلخيص العلاقات الدهمة بعد إجراء اختبار السببية في

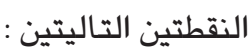

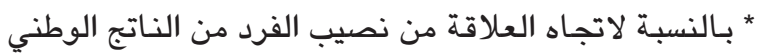

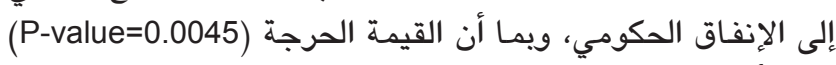

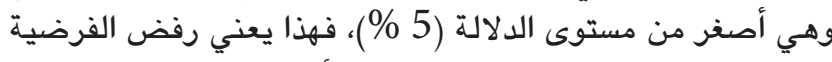

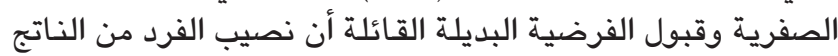

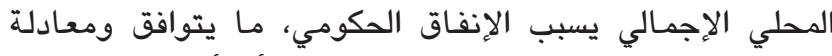
Musgrave

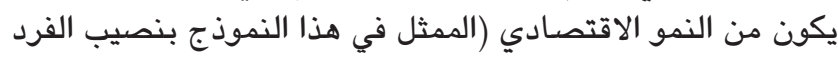

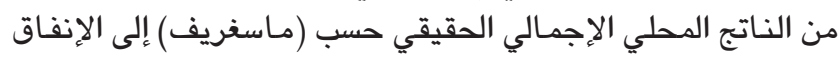

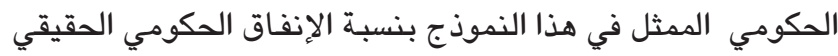

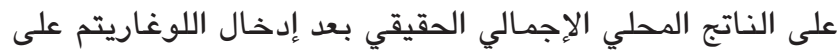

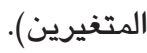

* بالنسبة لاتجاه العلاقة من الإنفاق الحكومي إلى النمو

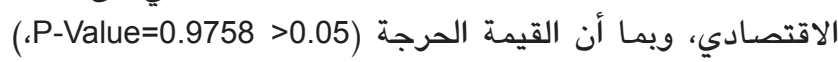

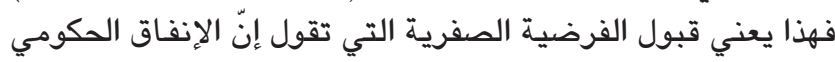

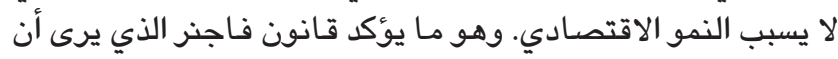

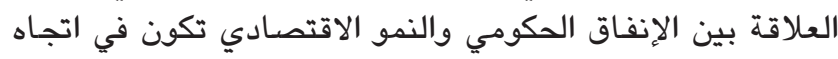

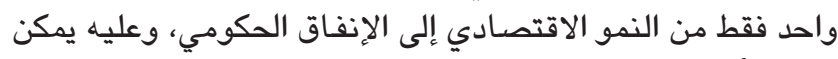

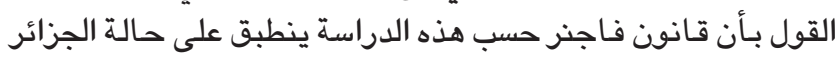
خلال الفترة الممتدة من 1967 - 2016.

5. التحليل الاقتصادي لمطابقة قانون (فاجنر) على الاقتصاد

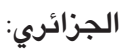

انتهجت الحكومة الجزائرية سياسة التوسع في الإنفاق

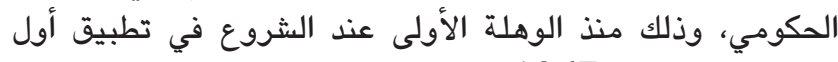

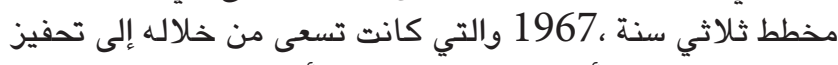

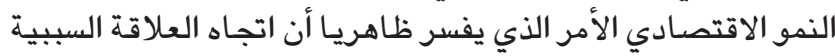

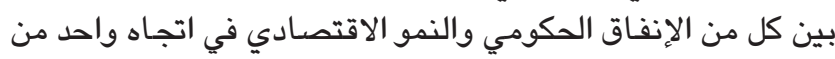


جامعة باتنة، الجزائر، (2015). بتول، مطر الجبوري، ودعاء، محمد الزاملي، دور الإنفاق الحكومي في

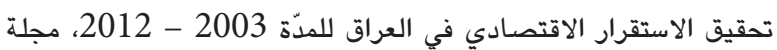

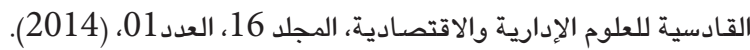
سالم عبد الله محمد باسويد، أثر الإنفاق الحكومي على النمو الاقتصادي

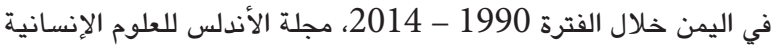
والاجتماعية، العدد 16، (2017). سمير، آيت يحيى، التحديات النقدية الدولية ونظام الصرف الملائم للجزائر، أطروحة دكتوراه، جامعة بـاتنة، الجزائر، (2014). ابن عزة، محمد، ترشيد سياسة الإنفاق العام باتباع منهج الانضباط

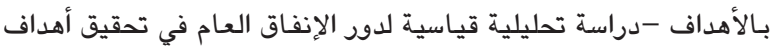

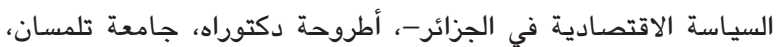

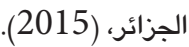
عمر محمود أبو عيدة، أثر الإنفاق الحكومي على النمو الاقتصادي في تلافي

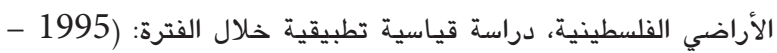

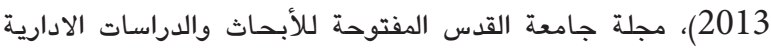

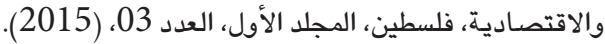
فتحي أحمد دياب عواد، اقتصاديات المالية العامة، دار الرضوان للنشر والتوزيع، عمان، (2013). كريم سالم حسين الغالبي، (الإنفاق الحكومي واختبار قانو فاجنر (Wagnerıs Law) الغري للعلوم الاقتصادية والإدارية، مجلة جامعة القادسية، العراق، المجلد 08، العدد 25، (2012). 10. كمال، شريط ، واقع الاستثمار الأجنبي وأثره على النمو الاقتصادي في

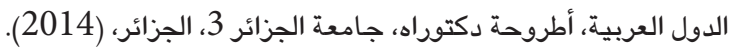
11. ليليا، غضابنة، العلاقة بين الإنفاق الحكومي والنمو الاقتصادي في

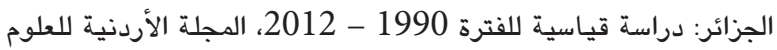
الاقتصادية، الهجلد 02، العدد 01، (2015).

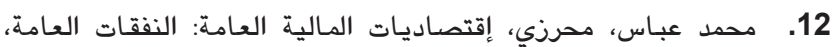

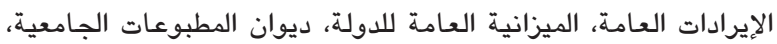

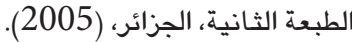
1، محمود، بيداري، العوامل المفسرة لنمو الإنفاق الحكومي في الاقتصاد

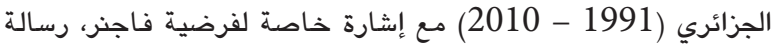
ماجستير، جامعة وهران، الجزائر، (2014) (2010). 14. 14. نور الدين، بوالكور، نمو الإنفاق الحكومي في الجزائر بين قانون

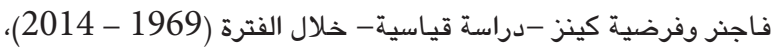
مجلة العلوم الإحصائية، العدد 08، (2017).

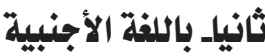

1. Abderrahim, C. and al., 2010, "The Macroeconomic Effects of fiscal policy shoks in Algeria: an empirical study», working paper series, $N$ 536, economic research forum.

2. Dickey, D. and W. Fuller, 1981, Likelihood Ratio Statistics for Autoregressive Time Series with a Unit Root», Econometrica, 49.

3. Engle, R.F. and C. Granger, 1987, Co-integration and Error Correction: Representation, Estimation and Testing, Econometrica 55, pp. 251-76. .3
ممثلا في حصة الإنفاق الحكومي من الناتج المحلي الإجمالي- عند الفارق الأول، كما أظهر النموذج وجود علاقئة من تكامل متزامنة بين

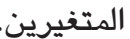

2. أظهر اختبار سببية (جرانجر) أنه توجد علاقة سبجية في المدى الطويل وفي اتجاه واحد من النمو الاقتصادي إلى الإنفاق إنهار الحكومي وهو ما يتوافق وقانون( فاجنر).

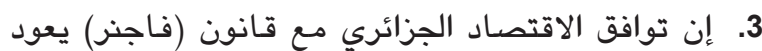
بالدرجة الأولى إلى أن الانفاق الحكومي في الجزائر هو متغير داخلي الحاني

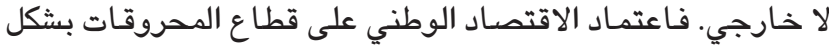

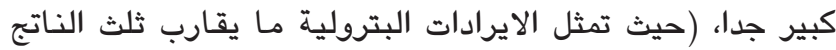

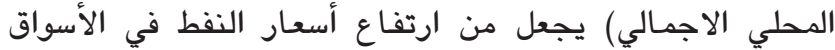

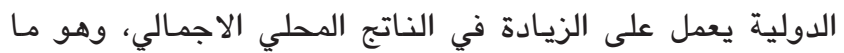

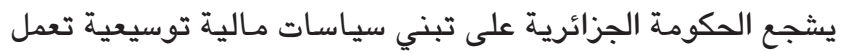
على الزيادة في الإنفاق الح كومي وليس العيس علينس.

4. بدأت الحكومة الجزائرية في اتباع سياسة مالية تقشفية ولية

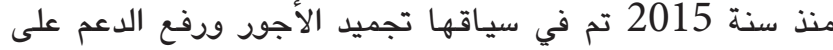
بعض السلع الضرورية ( كما حدث مع أسعار البنزين) وتجميد

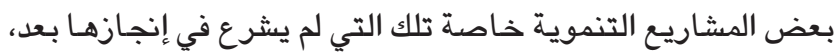

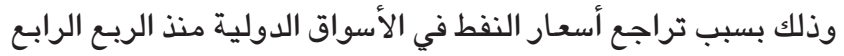

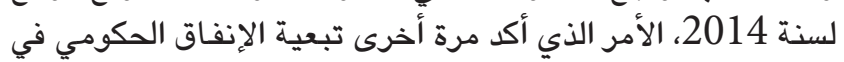
الجزائر للناتج المحلي الإجمالي. كما خلصت الدراسة إلى التوصيات الآتية:

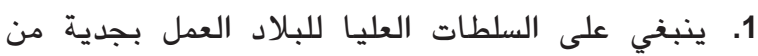
أجل تخليص الاقتصاد الوطني من تبعية المحروقات في تمويل

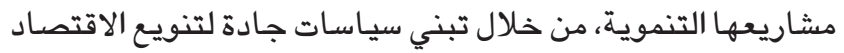
خارج القطاع الريعي.

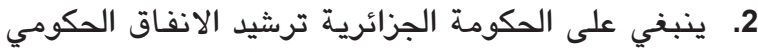

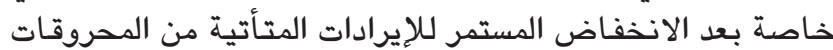

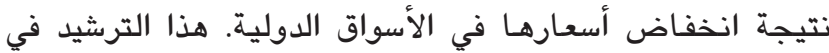

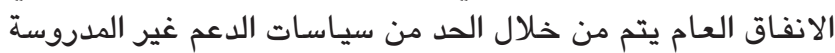

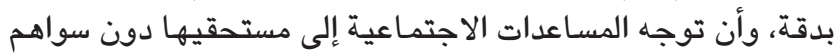
من طبقات المجتمع الميسورة، يضـاف إلى ذلك تطوير إدارة الرقابة الماتِ

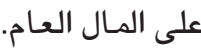

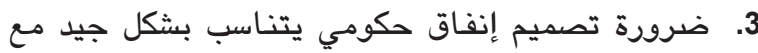

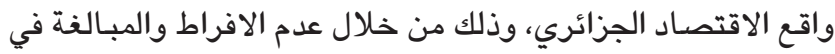

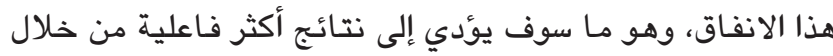
تحقيق معدلات نمو أعلى من تلك المحققة خلال السنوات السي ندات السابقة.

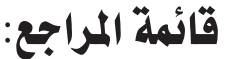

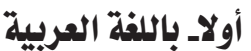

1. أشواق، بن قدور، تطور النظام المالي والنمو الاقتصادي، دراسة قياسية

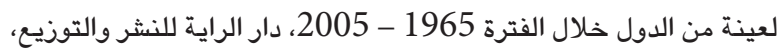

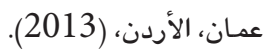

2. إيمان، بوعكاز، أثر الإنفاق العمومي على النمو الاقتصـادي دراسة قياسية

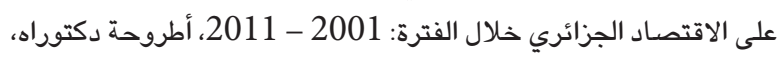


4. Fo-Kossi, E.T., 2018, Causality between Public Expenditures and Grwth in Togo, MPRA, Paper $n^{\circ} 87005$.

5. Gizen, V., et al., 2017, Public Expenditure and Economic Growth, Was Wagner Right? Evidence from Turkey, Academic Journal of Economic Studies, vol 03, $n^{\circ} 02$.

6. Granger, C.W., 1988, Some Recent Development in a concept of Causality, Journal of econometrics 39, pp. 144-211.

7. Monogbe, T.G. and J. Okaho, 2016, Government Spending and Economic GrowthPocess in Nigeria, Frantiers of accounting and Finance, vol 01, $n^{\circ} 01$.

8. Musgrave, R.A. and A.T. Peacock, eds., 1958, Classics in the theory of public finance (Macmillan, London).

9. Musgrave, R.A., 1969a, Cost-benefit analysis in the theory of public finance, Journal of Economic Literature 7.

10. Phillips, P.C.B. and P. Perron, 1988, Testing for a Unit Root in Times Series Regression, Biometrika 75, pp. 335-46. 